\title{
Rossby waves and two-dimensional turbulence in a large-scale zonal jet
}

\author{
Article \\ Published Version
}

Shepherd, T. G. (1987) Rossby waves and two-dimensional turbulence in a large-scale zonal jet. Journal of Fluid Mechanics, 183 (1). pp. 467-509. ISSN 0022-1120 doi: https://doi.org/10.1017/S0022112087002738 Available at https://centaur.reading.ac.uk/32992/

It is advisable to refer to the publisher's version if you intend to cite from the work. See Guidance on citing.

Published version at: http://dx.doi.org/10.1017/S0022112087002738

To link to this article DOI: http://dx.doi.org/10.1017/S0022112087002738

Publisher: Cambridge University Press

All outputs in CentAUR are protected by Intellectual Property Rights law, including copyright law. Copyright and IPR is retained by the creators or other copyright holders. Terms and conditions for use of this material are defined in the End User Agreement.

\section{www.reading.ac.uk/centaur}

\section{CentAUR}

Central Archive at the University of Reading

Reading's research outputs online 


\title{
Rossby waves and two-dimensional turbulence in a large-scale zonal jet
}

\author{
BY THEODORE G. SHEPHERD $\dagger$ \\ Center for Meteorology and Physical Oceanography, Massachusetts Institute of \\ Technology, Cambridge, MA 02139, USA
}

(Received 27 November 1985 and in revised form 10 February 1987)

The theory of homogeneous barotropic beta-plane turbulence is here extended to include effects arising from spatial inhomogeneity in the form of a zonal shear flow. Attention is restricted to the geophysically important case of zonal flows that are barotropically stable and are of larger scale than the resulting transient eddy field.

Because of the presumed scale separation, the disturbance enstrophy is approximately conserved in a fully nonlinear sense, and the (nonlinear) wavemean-flow interaction may be characterized as a shear-induced spectral transfer of disturbance enstrophy along lines of constant zonal wavenumber $k$. In this transfer the disturbance energy is generally not conserved. The nonlinear interactions between different disturbance components are turbulent for scales smaller than the inverse of Rhines's cascade-arrest scale $\kappa_{\beta} \equiv\left(\beta_{0} / 2 u_{\mathrm{rms}}\right)^{\frac{1}{2}}$, and in this regime their leading-order effect may be characterized as a tendency to spread the enstrophy (and energy) along contours of constant total wavenumber $\kappa \equiv\left(k^{2}+l^{2}\right)^{\frac{1}{2}}$. Insofar as this process of turbulent isotropization involves spectral transfer of disturbance enstrophy across lines of constant zonal wavenumber $k$, it can be readily distinguished from the shear-induced transfer which proceeds along them. However, an analysis in terms of total wavenumber $\kappa$ alone, which would be justified if the flow were homogeneous, would tend to mask the differences.

The foregoing theoretical ideas are tested by performing direct numerical simulation experiments. It is found that the picture of classical beta-plane turbulence is altered, through the effect of the large-scale zonal flow, in the following ways: (i) while the turbulence is still confined to $\kappa>\kappa_{\beta}$, the disturbance field penetrates to the largest scales of motion; (ii) the larger disturbance scales $\kappa<\kappa_{\beta}$ exhibit a tendency to meridional rather than zonal anisotropy, namely towards $v^{2}>u^{2}$ rather than vice versa; (iii) the initial spectral transfer rate away from an isotropic intermediate-scale source is significantly enhanced by the shear-induced transfer associated with straining by the zonal flow. This last effect occurs even when the large-scale shear appears weak to the energy-containing eddies, in the sense that $\mathrm{d} U / \mathrm{d} y \ll \kappa u_{\mathrm{rms}}$ for typical eddy length and velocity scales.

\section{Introduction}

By virtue of the vertical 'stiffness' imparted by rotation to a homogeneous fluid, the study of two-dimensional or barotropic systems has always played a central role in geophysical fluid dynamics. Although barotropic theory is not strictly applicable

$\dagger$ Present affiliation: Department of Applied Mathematics and Theoretical Physics, University of Cambridge, Silver Street, Cambridge, CB3 9EW, UK. 
to a stratified fluid, it is nevertheless thought to be qualitatively relevant in various ways - particularly for horizontal scales of motion larger than the internal Rossby radius of deformation - and it offers the compelling advantage of relative simplicity.

However the barotropic vorticity equation is itself analytically intractable because of advective nonlinearities, and on the energy-containing scales these nonlinearities usually cannot be ignored in geophysical applications. An idealization that has had some success, especially in meteorology, has been to treat the two-dimensional flow as if it were homogeneous turbulence. Using statistical methods and hypotheses that rely on the spatial and temporal homogeneity (Fjørtoft 1953; Kraichnan 1967; Leith 1968; Batchelor 1969), one can deduce that an intermediate-scale energy-enstrophy source will lead to spectral transfers of energy and of enstrophy respectively to larger and to smaller scales of motion. This prediction is qualitatively in accord with observed atmospheric behaviour (e.g. Chen \& Wiin-Nielsen 1978; Boer \& Shepherd 1983), with the intermediate-scale source identified with a forcing of the barotropic mode, at the scale of the Rossby deformation radius, by the process of baroclinic instability.

Nevertheless the atmosphere departs from the idealization of classical twodimensional turbulence theory in significant ways. Thus the theory has previously been extended to include the geophysical effects of Rossby-wave propagation (Rhines 1975; Holloway \& Hendershott 1977; Legras 1980), topography (Bretherton \& Haidvogel 1976; Herring 1977; Holloway 1978), weak horizontal divergence (Holloway 1983; Farge \& Sadourny 1986), and stratification (Charney 1971; Rhines 1977; Salmon 1978, 1980), but in all cases horizontal statistical homogeneity was imposed. Lin (1982) has recently treated the problem of two-dimensional turbulence in an inhomogeneous mean flow using closure methods, but the inhomogeneity was required to be extremely weak; it would seem that turbulent closure is impossibly cumbersome unless such a restriction is made. However, it is evident from observational data that the atmosphere is significantly inhomogeneous, at least on the larger scales of motion: in particular, the flow has a strong stationary and zonally symmetric component which varies substantially with latitude.

Observations reveal a separation in scale between this zonally symmetric stationary flow component and the energy-containing transient eddies (Boer \& Shepherd 1983). A reasonable first step for extending geostrophic turbulence into the inhomogeneous regime would therefore seem to be the investigation of two-dimensional beta-plane turbulence in the presence of a large-scale zonal shear flow. Such is the object of the present work. The beta-plane is probably the simplest system that can embrace the problem of inhomogeneous waves and turbulence, while still providing insight into large-scale geophysical fluid dynamics. McWilliams \& Chow (1981) have previously performed a numerical simulation study of a three-layer wind-driven channel model representing the Antarctic Circumpolar Current, with a mean flow that was strongly inhomogeneous. But there remain important questions to be addressed within the comparatively simpler barotropic context. Fortunately one can hope to capture some of the aspects of the behaviour of large-scale barotropic motions in a stratified, quasi-geostrophic fluid by using a barotropic model; this is because, at least within the context of baroclinic geostrophic turbulence theory (e.g. Rhines 1979; Salmon 1982; Sadourny 1985), the principal coupling between the barotropic and the gravest baroclinic modes occurs at a single scale - the Rossby deformation radius.

The governing equation is presented and discussed in \$2. It is then argued (\$3.1) that the flow dynamics is most easily understood by focusing attention on two quite distinct, though strongly coupled, processes. The first of these is the interaction 
between the large-scale zonal flow and non-zonal disturbances of smaller scale: this interaction can always be viewed to a good approximation, even at finite amplitude, as a shear-induced spectral transfer of disturbance enstrophy along lines of constant zonal wavenumber $k(\$ 3.1)$. In such transfer the disturbance energy is generally not conserved. This process is studied in detail in the context of linearized theory, using a spectral version of ray-tracing theory (\$3.2).

The second key element of the dynamics is the interaction between different disturbance components : a principal effect of this process is to make the disturbance field locally isotropic by spreading the enstrophy along curves of constant total (two-dimensional) wavenumber $\kappa(\$ 3.3)$. In particular, isotropization involves spectral transfer across lines of constant zonal wavenumber $k$, and thus has effects quite distinct from the first process of shear-induced spectral transfer along lines of constant $k$. A spectral analysis in terms of $\kappa$ alone would tend to obscure the distinction. $\dagger$ Perhaps needless to say, the disturbance self-interactions can also be anticipated to yield spectral transfer to larger and to smaller $\kappa$ in the usual way; but the timescale of such transfer is slower than that of turbulent isotropization.

The validity of the separate identification of the two processes, and the way in which they interact, are examined by considering various direct numerical simulations in §4. This study does not attempt a thorough investigation of parameter space; rather, the non-dimensional parameters are chosen to represent observed conditions in the troposphere. This indeed falls within a regime in which both nonlinearity and inhomogeneity are significant, even though the large-scale time- and zonally averaged shear would appear weak to the energy-containing eddies in a scale analysis.

The theoretical and numerical results are discussed in $\$ 5$, together with their implications for transient eddy parameterization in atmospheric models. Some parameter dependences are also described. It is noted in addition that the zonal jets generated internally in classical beta-plane turbulence simulations do not induce the phenomena found here; the reasons for this are also discussed in $\S 5$.

\section{Mathematical description}

\subsection{Equations for a disturbed flow}

The governing equation for the barotropic beta-plane is

$$
\nabla^{2} \psi_{t}+\mathrm{J}\left(\psi, \nabla^{2} \psi\right)+\beta_{0} \psi_{x}=\mathrm{S}(\psi)
$$

where $\psi$ is the stream function, $\mathrm{J}$ is the two-dimensional Jacobian, $\beta_{0}$ is the (constant) planetary vorticity gradient, $S$ is a linear operator representing all source and sink terms, including viscosity, and the other symbols have their usual meanings. It is convenient to consider (2.1) over a doubly periodic domain.

If the statistics of the flow field are independent of spatial position, so that the flow is spatially homogeneous, then (2.1) describes classical two-dimensional betaplane turbulence. The characteristics of such a flow are now well known (see e.g. Rhines 1975, 1979): the domain-integrated energy $\frac{1}{2}|\nabla \psi|^{2}$ and enstrophy $\frac{1}{2}\left(\nabla^{2} \psi\right)^{2}$ are invariant for conservative flow $(S=0)$, and these two inviscid constraints, together with a statistical assumption giving an 'arrow of time' - such as the broadening of

$\dagger$ Of course turbulent interactions involve transfer along $k$-lines too, though this transfer will not be enstrophy-conserving for each $k$. The essential difference between classical homogeneous two-dimensional turbulence and the shear-induced transfer discussed here is that the straining field of the latter is not considered to be random, and so some phase information must be retained. 
spectral lines (Batchelor 1953, p. 186), or more generally the increase of a suitable measure of flow entropy (Carnevale 1982) - imply a net nonlinear transfer of energy to larger scales, and of enstrophy to smaller scales (Fjørtoft 1953; Merilees \& Warn 1975). The up-scale 'cascade' is arrested when Rossby-wave dispersion takes over from nonlinear advection, at a scale $\kappa_{\beta}$ whose magnitude is suggested by simple scaling analysis (Rhines 1975; Holloway \& Hendershott 1977). From then on the development is characterized by a slow evolution into quasi-zonal jets (Rhines 1975; Holloway \& Hendershott 1977). A forced-dissipative equilibrium model exhibiting these features would have an energy-enstrophy input at some intermediate scale, together with a highly scale-selective diffusion operator to remove the cascading enstrophy at small scales, and perhaps a scale-independent Ekman drag term to prevent energy from accumulating at large scales. Numerical experiments of this nature have been performed recently by, among others, Basdevant et al. (1981).

The problem of present interest, however, is that of beta-plane turbulence in the presence of an inhomogeneous steady basic zonal flow $\Psi(y)$. Such a basic flow is itself a solution of $(2.1)$ with $S(\Psi)=0$. Writing the full stream function as

$$
\psi=\Psi+\phi
$$

the 'disturbance' $\phi$ must satisfy the (exact) equation

$$
\nabla^{2} \phi_{t}+\mathrm{J}\left(\phi, \nabla^{2} \phi\right)+\mathrm{J}\left(\Psi, \nabla^{2} \phi\right)+\mathrm{J}\left(\phi, \nabla^{2} \Psi\right)+\beta_{0} \phi_{x}=\mathrm{S}(\phi) .
$$

One may define the disturbance energy as

$$
E(\phi)=\iint \frac{1}{2}|\nabla \phi|^{2} \mathrm{~d} x \mathrm{~d} y=\iint \frac{1}{2}\left(u^{2}+v^{2}\right) \mathrm{d} x \mathrm{~d} y,
$$

where $u \equiv-\phi_{y}$ and $v \equiv \phi_{x}$. The disturbance-energy equation is obtained by multiplying (2.3) by $\phi$ and integrating the result over the doubly periodic domain. When $\mathrm{S}(\phi)=0$ this yields

$$
\begin{aligned}
\frac{\mathrm{d}}{\mathrm{d} t} \iint \frac{1}{2}|\nabla \phi|^{2} \mathrm{~d} x \mathrm{~d} y & =\iint \phi \mathrm{J}\left(\Psi, \nabla^{2} \phi\right) \mathrm{d} x \mathrm{~d} y=-\iint \Psi \mathrm{J}\left(\phi, \nabla^{2} \phi\right) \mathrm{d} x \mathrm{~d} y \\
& =\iint \nabla^{2} \phi \mathrm{J}(\phi, \Psi) \mathrm{d} x \mathrm{~d} y \\
& =\iint U \frac{\partial(u v)}{\partial y} \mathrm{~d} x \mathrm{~d} y=-\iint(u v) \frac{\mathrm{d} U}{\mathrm{~d} y} \mathrm{~d} x \mathrm{~d} y
\end{aligned}
$$

where $U \equiv-\Psi_{y}$. While $E(\phi)$ is called the disturbance energy, in conformity with common usage, we recall the well-known fact that the basic-flow and disturbance energies are not additive (cf. Möhring 1978; Held 1985). Indeed, as (2.5) shows, even when $S=0$ (and so the total energy $E(\psi)$ is conserved), $E(\phi)$ is not conserved because of the $J\left(\Psi, \nabla^{2} \phi\right)$-term in $(2.3)$, representing straining of the disturbance vorticity by the shear in the basic flow.

To obtain the disturbance-enstrophy equation, multiply (2.3) by $\nabla^{2} \phi$. Then integrating the expression over the domain yields the equivalent to $(2.5 a, b)$, namely

$$
\begin{aligned}
\frac{\mathrm{d}}{\mathrm{d} t} \iint \frac{1}{2}\left(\nabla^{2} \phi\right)^{2} \mathrm{~d} x \mathrm{~d} y & =-\iint \nabla^{2} \phi \mathrm{J}\left(\phi, \nabla^{2} \Psi\right) \mathrm{d} x \mathrm{~d} y \\
& =\iint \nabla^{2} \Psi \mathrm{J}\left(\phi, \nabla^{2} \phi\right) \mathrm{d} x \mathrm{~d} y,
\end{aligned}
$$


but here it is the $\mathrm{J}\left(\phi, \nabla^{2} \Psi\right)$-term in (2.3) that violates conservation of disturbance enstrophy. For a barotropic fluid, this term is similar to enstrophy generation by topography (although topography does not enter into the energy balance and $\Psi$ does).

\subsection{Scaling analysis}

Considering now (2.3) with $\mathbf{S}=\mathbf{0}$, non-dimensionalize by scaling the terms with $\Psi=U_{0} L_{0} \Psi^{\prime}$ for the basic flow, and $\phi=u_{0} \lambda_{0} \phi^{\prime}$ for the disturbance. The primed quantities are taken to be order unity. Let $t=T t^{\prime}$, with $T$ to be determined. Then the non-dimensional equation, after dropping the primes, is

$$
\left(\frac{\lambda_{0}}{u_{0} T}\right) \nabla^{2} \phi_{t}+\mathrm{J}\left(\phi, \nabla^{2} \phi\right)+\alpha \mathrm{J}\left(\Psi, \nabla^{2} \phi\right)+\alpha \gamma^{2} \mathrm{~J}\left(\phi, \nabla^{2} \Psi\right)+\beta \phi_{x}=0
$$

where the following non-dimensional parameters have been introduced:

$$
\alpha \equiv \frac{U_{0}}{u_{0}} ; \quad \gamma \equiv \frac{\lambda_{0}}{L_{0}} ; \quad \beta \equiv \frac{\beta_{0} \lambda_{0}^{2}}{u_{0}} .
$$

It may be recalled that the arrest of the up-scale energy cascade in classical beta-plane turbulence (Rhines 1975) occurs at a scale where $\beta \approx 1$, equivalently at a wavenumber

$$
\kappa_{\beta} \approx\left(\frac{\beta_{0}}{2 u_{0}}\right)^{\frac{1}{2}}
$$

(the factor of two arising from an assumption that the zonal and meridional scales are the same). $\dagger$ Note also that for the geophysically important case of a scale separation between the basic flow and the disturbance, which is indeed the case of interest in this study, one may take $\gamma \ll 1$; then the disturbance enstrophy is approximately conserved.

In (2.7), the ratio of linear to nonlinear effects is rather more complicated than the inverse wave steepness $\beta$ which is relevant to beta-plane turbulence (Rhines 1975; Shepherd $1987 a$ ); here it may be expressed as

$$
M \equiv \frac{\beta_{0} \lambda_{0}^{2}+U_{0}\left(\lambda_{0} / L_{0}\right)^{2}+U_{0}}{u_{0}} \equiv \beta+\alpha\left(\gamma^{2}+1\right) .
$$

While it is true that $M \ll 1$ still represents a highly nonlinear regime where the predictions of classical two-dimensional turbulence theory may be expected to hold, it is evident that the dynamics of the quasi-linear regime $M \gg 1$ is a good deal richer and much less tractable than that of the pure Rossby-wave regime of beta-plane turbulence. Note that if $\alpha \gg 1$, then $M \geqslant 1$ over all scales. In addition the combined spectral wave-turbulence 'jigsaw puzzle' is complicated by the fact that the disturbance energy and enstrophy are not generally conserved. However, it must be cautioned that this kind of scale analysis is very crude, and is particularly dangerous with regard to Jacobian terms. This is not a trivial matter, as Jacobians can yield resultants well short of their maximum value if the gradients of the two fields are nearly parallel; moreover the net energetic interaction between $\Psi$ and $\phi$ at a given time will vanish if $\phi$ is exactly isotropic (Lorenz 1953; Pedlosky 1962), despite the fact that $J\left(\Psi, \nabla^{2} \phi\right)$ is locally non-vanishing.

$\dagger$ Holloway \& Hendershott (1977) argue for the alternative $\kappa_{\beta} \approx \beta_{0} / \zeta_{\text {rms }}$, where $\zeta_{\mathrm{rms}}$ is the r.m.s. vorticity, which probably is indeed more appropriate in the forced-dissipative context with a broad equilibrium spectrum; but when the forcing is close to $\kappa_{\beta}$, as here, the two estimates are virtually the same. 


\subsection{Spectral diagnostic formulae}

The disturbance energy associated with wavenumber $\kappa=(k, l)$ is given by

$$
E_{\mathrm{D}}(\boldsymbol{\kappa}) \equiv \frac{1}{2} \kappa^{2}|\hat{\phi}(\boldsymbol{\kappa})|^{2},
$$

and may be broken up into its zonal and meridional components as follows:

$$
E_{\mathrm{D}}(\boldsymbol{\kappa})=\frac{1}{2} l^{2}|\hat{\phi}(\boldsymbol{\kappa})|^{2}+\frac{1}{2} k^{2}|\hat{\phi}(\boldsymbol{\kappa})|^{2}=\frac{1}{2}\left\{u^{2}\right\}(\boldsymbol{\kappa})+\frac{1}{2}\left\{v^{2}\right\}(\boldsymbol{\kappa}) .
$$

Here the curly brackets as well as the circumflex indicate a Fourier transform, and $k$ and $l$ are the $x$ - and $y$-wavenumbers. To obtain the energy equation for each $\boldsymbol{\kappa}$, take a Fourier transform of $(2.3)$, multiply the result by $\hat{\phi}^{*}(\boldsymbol{\kappa})$, and add the complex conjugate; this yields

$$
\frac{\partial}{\partial t} E_{\mathbf{D}}(\boldsymbol{\kappa})=I_{\mathbf{D}}(\boldsymbol{\kappa})+C_{\mathbf{D}}(\boldsymbol{\kappa})+D_{\mathbf{D}}(\boldsymbol{\kappa}) .
$$

$I_{\mathrm{D}}(\boldsymbol{\kappa})$ represents the energy transfer to $\boldsymbol{\kappa}$ arising from nonlinear interactions with other disturbance components, $C_{\mathrm{D}}(\kappa)$ that arising from interactions involving the basic-flow and disturbance components together. $D_{\mathrm{D}}(\kappa)$ represents explicit sourcesink effects. The three terms are respectively associated with the quantities $J\left(\phi, \nabla^{2} \phi\right), J\left(\Psi, \nabla^{2} \phi\right)+J\left(\phi, \nabla^{2} \Psi\right)$, and $\mathrm{S}$ in $(2.3)$, and are defined explicitly in the Appendix. The term arising from $\beta_{0}$ has not been included as it does not affect the energetics. The enstrophy-budget equation is similarly obtained through multiplying the Fourier transform of $(2.3)$ by $\left\{\nabla^{2} \phi\right\}^{*}(\kappa) \equiv-\kappa^{2} \phi^{*}(\kappa)$, and adding the complex conjugate, which yields just $\kappa^{2}$ times $(2.13)$ :

$$
\frac{\partial}{\partial t} G_{\mathrm{D}}(\kappa) \equiv \frac{\partial}{\partial t} \kappa^{2} E_{\mathrm{D}}(\kappa)=\kappa^{2} I_{\mathrm{D}}(\kappa)+\kappa^{2} C_{\mathrm{D}}(\kappa)+\kappa^{2} D_{\mathrm{D}}(\kappa) .
$$

The symbol $I$ is used here to denote a nonlinear interaction term, in the sense that it only redistributes the relevant quantity (in the cases of (2.13) and (2.14), respectively the disturbance energy and enstrophy) between different wavenumbers; hence

$$
\sum_{\boldsymbol{\kappa}} I_{\mathrm{D}}(\boldsymbol{\kappa})=\iint \phi \mathrm{J}\left(\phi, \nabla^{2} \phi\right) \mathrm{d} x \mathrm{~d} y=0
$$

and

$$
\sum_{\kappa} \kappa^{2} I_{\mathrm{D}}(\kappa)=-\iint \nabla^{2} \phi \mathrm{J}\left(\phi, \nabla^{2} \phi\right) \mathrm{d} x \mathrm{~d} y=0 .
$$

On the other hand $C$ denotes a nonlinear conversion term, arising from the nonlinear interaction with the basic flow, whose integrated effect is a net source or sink for the disturbance; some of the physical-space expressions for $C_{\mathrm{D}}$ and $\kappa^{2} C_{\mathrm{D}}$ were considered in (2.5) and (2.6). Of course, both $C_{\mathrm{D}}(\boldsymbol{\kappa})$ and $\kappa^{2} C_{\mathrm{D}}(\boldsymbol{\kappa})$ represent a combination of nonlinear disturbance interaction and basic-flow disturbance conversion, with the partition between these two processes being ambiguous at any given wavenumber.

For the interpretation of some of the numerical experiments, analogous spectral diagnostics describing a stationary-transient decomposition of the flow will be required. These are defined where they are needed, in $\$ 4.5$. 


\section{Theory}

\subsection{A spectral view of strongly nonlinear wave-mean-zonal-flow interaction}

If the nonlinear (advective) interactions between a zonal basic flow and an arbitrary disturbance are spectrally decomposed into wavevector 'triads' (cf. Lorenz 1960), then it is immediately obvious that these interactions are restricted by a very simple yet powerful geometrical constraint: the basic flow couples disturbance components of the same zonal wavenumber $k$. This property is depicted in figure 1 for the special case where the basic flow consists of a single mode $(k, l)=\left(0, l_{0}\right)$, in which a disturbance component $(k, l)$ interacts via the basic flow only with modes $\left(k, l+l_{0}\right)$ and $\left(k, l-l_{0}\right)$. When the basic flow consists of a spectrum of meridional modes then the same restriction applies, but now $(k, l)$ is coupled with a spectrum of modes of the same $k$, the localness of which will be determined by the localness of the spectral representation of the basic flow $\Psi$.

Now, it is equally obvious that this spectral constraint applies not only to the basic-flow disturbance interaction, but also - provided the physical problem, including any forcing, is zonally homogeneous - to the interaction between an equilibrated zonal flow and the deviations therefrom; in particular it would apply to the stationary and transient flow components in a forced-dissipative problem taken to statistical equilibrium.

It is worth pointing out that in spherical geometry, although nonlinear interactions are no longer confined to pairs of wavevector triads (e.g. Tang \& Orszag 1978), it is nevertheless true that an interaction between a zonal flow and a zonal $(k \neq 0)$ mode involves only modes with the same zonal wavenumber $k$. Consequently this constraint is likely to be useful in interpreting the spectral signature of stationary-transient interaction in the atmosphere (Shepherd $1987 b, c$ ).

If one now has a conservation law governing a disturbance quantity in the interaction between a zonal basic flow (or, depending on which problem is of interest, a mean flow, to which the comments of this section apply equally) and an arbitrary disturbance, then the above considerations suggest that it may be useful to characterize the interaction as a 'shear-induced spectral transfer' of that conserved disturbance quantity along lines of constant zonal wavenumber $k$. In this transfer process the basic flow, through its shear, plays an essential catalytic role in that it forms the third member of the triad, but it is passive in terms of the relevant disturbance 'energetics' (defined according to the conserved quantity, not the actual energy). This concept is a familiar one to the oceanic-internal-wave community, where the notion of 'induced diffusion' of wave action through highly non-local resonant triads is well established (e.g. Phillips 1977, §5.5). However in the present case there is no restriction to resonant interactions, and the formalism is, at least in principle, exact.

When the basic flow possesses either spatial or temporal symmetry, then a conservation theorem can always be found for the (linear) interaction between the basic flow and an arbitrary disturbance. In the former case the relation obtained is a small-amplitude form of 'pseudomomentum' (or, as some authors prefer, 'quasimomentum') conservation (Andrews \& McIntyre 1976; Held 1985), while in the latter case the relevant quantity is a small-amplitude form of 'pseudoenergy' (Andrews 1983). The difficulty is that, in general, these quantities are not conserved by the disturbance self-interactions, and thus fail to lead to fully nonlinear descriptions of the wave-mean-flow interaction.

In fact an examination of (2.5a) and (2.6b) immediately reveals that a fully 


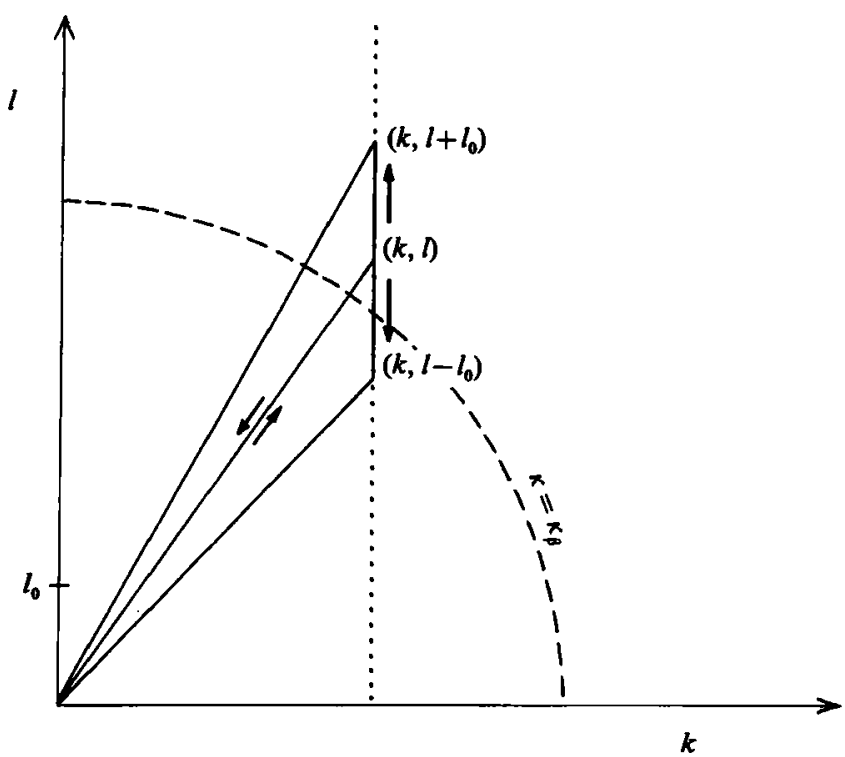

Figure 1. Possible triad interactions between a basic flow $\left(0, l_{0}\right)$ and an arbitrary disturbance component $(k, l)$, represented in terms of positive wavenumbers. Arrows indicate direction of energy transfers if the disturbance is to spread. The basic flow couples disturbances along the dotted vertical line of constant $k$. There is a possibility that this interaction mechanism may enable the disturbance to penetrate the 'Rhines radius' $\kappa=\kappa_{\beta}$.

nonlinear conservation theorem exists for the special case $\nabla^{2} \Psi=-c \Psi$ (for some constant $c$ ), in which the conserved disturbance quantity ((energy) $-c^{-1}$ (enstrophy)) (which may be written in the notation of $\$ 2.3$ as $E_{\mathrm{D}}-c^{-1} G_{\mathrm{D}}$ ) has a straightforward spectral representation: its $\kappa$-component is simply $\left(1-\left(\kappa^{2} / c\right)\right) E_{\mathrm{D}}(\boldsymbol{\kappa})$. Basic flows satisfying this linear functional relationship include those consisting of a single Fourier mode $\kappa_{0}$, in which case $c=\kappa_{0}^{2}$. (On the beta-plane, such a solution is made to satisfy $\nabla^{2} \Psi+\beta_{0} y=-c \Psi$ by adding a (vorticity-free) constant zonal flow $U=-\beta_{0} / c$ to $\Psi$.)

Although the basic flows to be considered in this study are of the particular form described above, the time-mean equilibrated flows (where appropriate) generally are not; moreover it would hardly be prudent to develop a theory applicable only in such restrictive circumstances. Instead, the presumed scale separation (which is a far more robust characteristic) will be exploited by noting that, as was mentioned in $\$ 2.2$, the disturbance enstrophy $G_{\mathrm{D}}$ is approximately conserved when $\gamma \ll 1$ (with $\gamma$ given in (2.8)). Indeed this is a natural limit of the previous special case when $\kappa_{0}^{2} \ll \kappa^{2}$.

The above discussion suggests that, to a first approximation, one may legitimately view the interaction between a large-scale zonal flow and an arbitrary disturbance as a shear-induced spectral transfer of disturbance enstrophy $G_{\mathbf{D}}$ along lines of constant zonal wavenumber $k$; this process would show up in the term $\kappa^{2} C_{\mathrm{D}}(k ; l)$ of (2.14), considered as a function of $l$ for fixed $k$. If one knows the transfer of enstrophy, moreover, this immediately yields the sense of the energy conversion: induced up-scale enstrophy transfer requires a net conversion of energy from the large-scale flow to the disturbance, down-scale enstrophy transfer the opposite. Viewed in terms of $G_{\mathrm{D}}$, namely by examining $\kappa^{2} C_{\mathrm{D}}(k ; l)$, the wave, mean-flow interaction is spectrally 'local' insofar as it consists of transfers over small ranges of wavenumbers. From an energetic standpoint, however, the process is fundamentally non-local in that it 
involves non-negligible transfers between the large-scale flow and the disturbance (the associated non-local enstrophy transfers being present but negligible).

The final essential property of $G_{\mathrm{D}}$ that is relevant here is its conservation in interactions between various disturbance flow components, that is to say in the 'wave-wave' interactions represented by $\kappa^{2} I_{\mathrm{D}}(\boldsymbol{\kappa})$ of (2.14). It is this property that makes $G_{\mathrm{D}}$ such a useful diagnostic quantity for investigating strongly nonlinear wave-mean-flow interaction. In principle $G_{\mathrm{D}}(\boldsymbol{x})$ can move freely over the spectral domain via disturbance self-interactions, in particular - in contrast to the previous class of interactions - across lines of constant $k$. In fact, both the disturbance energy $E_{\mathrm{D}}$ and $G_{\mathrm{D}}$ are conserved in these interactions, as expressed by $(2.15 a, b)$; indeed this constraint proves essential in understanding the enstrophy transfers associated with the nonlinear dynamics of the problem, as will be seen in $§ 3.3$.

\subsection{Ray tracing in a zonal jet}

To understand the nature of the shear-induced spectral transfer discussed above, it is reasonable to begin by looking at the 'linear' interaction between a zonal jet and an arbitrary disturbance, where linear in this context means that the zonal flow is held fixed. For a significant scale separation, i.e. $\gamma \ll 1$, this interaction is primarily characterized as a shearing of disturbance vorticity by the large-scale zonal flow, via the $J\left(\Psi, \nabla^{2} \phi\right)$-term of (2.3). In fact this process is so ubiquitous and so central to the dynamics that it is worth considering it in the context of an important special case before proceeding to a more general treatment.

When the basic flow takes the special form of linear Couette flow $U(y) \propto y$ (this does not satisfy the periodic boundary conditions of the present problem, but no matter), and $\beta=0$, then the linearized version of the vorticity equation (2.3) reduces, for a suitably chosen timescale, to

$$
\left(\frac{\partial}{\partial t}+y \frac{\partial}{\partial x}\right) \nabla^{2} \phi=0
$$

this equation allows an exact solution, as shown long ago by Kelvin (Thomson 1887). Avoiding the question of boundary effects, (3.1) describes a clockwise tilting about $y=0$ of disturbance wavecrests (figure 2). Since Fourier modes provide a complete representation of any smooth initial condition, the problem is thus solved. The key points to be noted from figure 2 are that the zonal wavenumber $k$ is fixed, while the meridional wavenumber $l$ is continuously varying; moreover the wave enstrophy is conserved. If the wavecrests initially lean into the mean shear (figure $2 a$ ) then $|l|$ decreases, passes through zero, and then increases without limit; the first stage corresponds to an amplification of the wave energy density, as first discussed by Orr (1907). If, however, the wavecrests are initially vertical (figure $2 b$ ) or lean along the mean shear (figure $2 c$ ), then $|l|$ simply increases according to $|l|=\left|l_{1}\right|+\left|k_{1}\right|\left(t-t_{1}\right)$. In both cases the end state is $|l| \rightarrow \infty$, or a zonal orientation of the crests. An energetic argument based on a consideration of the Reynolds stress would indicate that the wave energy is then entirely transferred to the zonal flow.

It is to be noted that the process just described conforms to the spectral constraints discussed in \$3.1, but goes further in providing a solution to the linearized problem. Furthermore, it is evident that the inclusion of non-zero $\beta$ makes no difference to the result, as a propagating wave will everywhere encounter the same basic shear. (On the other hand Rossby-wave dispersion can affect the local energy density of an inhomogeneous sea of wave packets.)

For a general zonal flow with variable shear, however, no exact analytic solution 

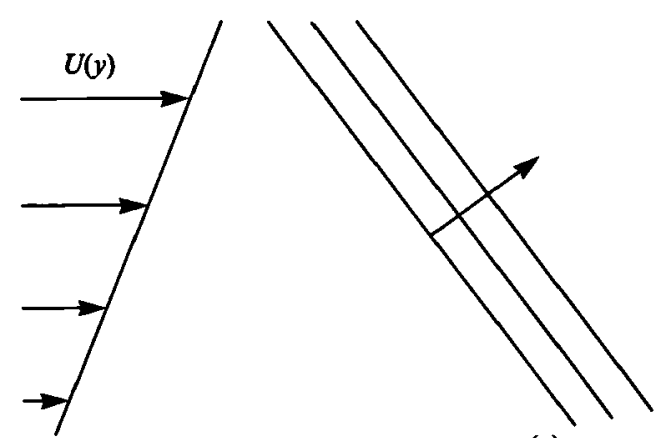

(a)

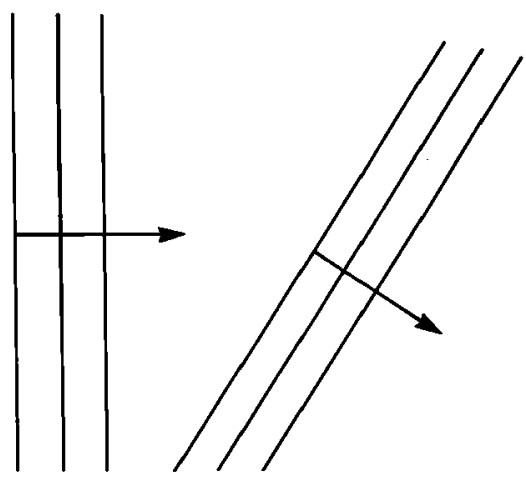

(b)

(c)

Fiadre 2. Advective shearing of plane wavecrests by linear Couette flow, in a time sequence from left to right. Arrows denote wavevectors.

has yet been found. Fortunately such inhomogeneous problems can be treated approximately using ray-tracing theory (see e.g. Lighthill $1978, \$ 4.6$ for more details) when the basic state varies slowly over a wavelength of the disturbance. The condition of slow basic-state variation means that solutions may be sought in the form of 'wave packets': namely, localized disturbance envelopes modulating a dominant wavenumber $\boldsymbol{\kappa}=(k, l)$. Such wave-packet solutions propagate on the basic state according to the 'ray-tracing' equations

$$
\frac{\mathrm{d} x}{\mathrm{~d} t}=\frac{\partial \omega}{\partial k}=U+\frac{\partial \hat{\omega}}{\partial k}, \quad \frac{\mathrm{d} y}{\mathrm{~d} t}=\frac{\partial \omega}{\partial l}=\frac{\partial \hat{\omega}}{\partial l} ;
$$

while the wavenumber evolves according to the generalized Snell's law

$$
\frac{\mathrm{d} k}{\mathrm{~d} t}=-\frac{\partial \omega}{\partial x}=0, \quad \frac{\mathrm{d} l}{\mathrm{~d} t}=-\frac{\partial \omega}{\partial y}=-k \frac{\mathrm{d} U}{\mathrm{~d} y},
$$

the derivative $\mathrm{d} / \mathrm{d} t$ in (3.3) referring to the Lagrangian derivative evaluated along the ray (3.2). In the above the 'intrinsic frequency' $\hat{\omega}$ is given by the Rossby-wave dispersion relation

$$
\hat{\omega}(k, l, x, y)=-\frac{\beta_{0} k}{k^{2}+l^{2}},
$$

and the absolute frequency $\omega=\hat{\omega}+U k$ for a zonal basic flow. All quantities are at most slowly varying functions of $y$. Equation (3.3a) expresses the fact that conservation of $k$ is a direct consequence of zonal homogeneity; similarly, the time invariance of the mean flow incorporated implicitly in (3.3) implies conservation of $\omega$ (along the ray path): $\mathrm{d} \omega / \mathrm{d} t=0$. To find the change in wave energy $E$ along the ray, one may appeal to the fact that the integrated wave action $A=E / \hat{\omega}$ is conserved following the packet (Bretherton \& Garrett 1968).

The connection between the approximate theory of ray tracing and the exact solution for Couette flow is made clear by a consideration of the wave-packet dynamics. Choosing a positive shear and $k>0$ for definiteness, imagine two wave packets as shown in figure 3: packet $A$ has $k / l>0$, or crests leaning into the basic shear; while packet $B$ has $k / l<0$, or crests leaning along the shear. Consider first packet $A$. By (3.4) and (3.2b), it has a northward or positive meridional group 

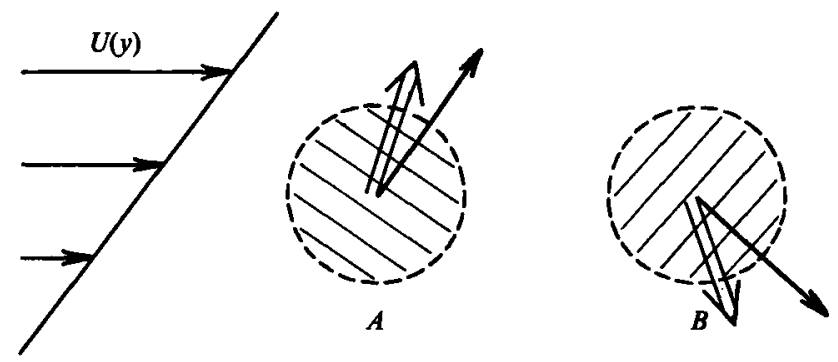

Figure 3. Propagation of two wave packets $A$ and $B$ in shear flow. Lines show wavecrests, single arrows wavevectors, and double arrows the group-velocity vectors.

velocity $\partial \omega / \partial l$; as it propagates, however, its crests are tilted by the basic shear as in figure $2(a, b)$, and this is reflected in the decreasing value of $|l|$ predicted by (3.3b). As in the constant-shear case, $k$ is fixed (see $(3.3 a)$ ). The orientation of the crests is such that the associated Reynolds stress extracts energy from the zonal flow, and this is again consistent with the ray-tracing picture: (3.4) implies that if the total wavenumber decreases, then $|\hat{\omega}|$ increases; thus conservation of wave action implies an increase in wave energy, an increase that will be especially pronounced for small $k$.

Similar reasoning applied to packet $B$, which has $l<0$, demonstrates that it propagates southward, $l$ becomes more negative, hence $|\hat{\omega}|$ and the wave energy decrease. The general result is that packets propagating into increasingly westerly flow increase both their wave energy and their meridional wavelength, while packets propagating into increasingly easterly flow decrease both quantities.

Since the ratio $\left\langle u^{2}\right\rangle /\left\langle v^{2}\right\rangle$ of zonal to meridional energy $(\langle$.$\rangle representing a phase$ average) for each packet is given by $l^{2} / k^{2}$ (cf. (2.12)), it follows that this ratio decreases for packet $A$ and increases for packet $B$. More generally, disturbances (like packet $A)$ evolving to larger scale become increasingly meridionally anisotropic, while disturbances (like packet $B$ ) evolving to smaller scale become increasingly zonally anisotropic.

A timescale for this process of shear-induced spectral transfer can be determined as follows. If $\tau$ is the e-folding time for the spatial scale of the disturbance, then

$$
\frac{\kappa}{\tau}=\left|\frac{\mathrm{d} \kappa}{\mathrm{d} t}\right|=\left|\frac{l}{\kappa} \frac{\mathrm{d} l}{\mathrm{~d} t}\right|=\left|\frac{k l}{\kappa} \frac{\mathrm{d} U}{\mathrm{~d} y}\right| \Leftrightarrow \tau=\frac{k^{2}+l^{2}}{|k l \mathrm{~d} U / \mathrm{d} y|} .
$$

At fixed $k$ (and $y$ ), $\tau$ is minimized for $k^{2}=l^{2}$, and it increases rapidly with $|l|$ when $|l|$ is large.

It is now possible to construct the solution to the spectral transfer problem of $\$ 3.1$ that is provided by ray-tracing theory. Given spectrally symmetric initial conditions, namely a packet with $k / l<0$ matching each with $k / l>0$, with equal amplitude and the same starting latitude $y_{i}$, then each pair of packets will propagate initially in opposite meridional directions with equal speeds, as in figure 3 ; in the spectral domain this will appear as a pair of enstrophy 'pulses' of equal amplitude, moving one to larger and one to smaller $|l|$ but at the same rate, along a line of constant $k$. As a consequence of the relation between enstrophy and energy, this latter picture will be represented in energetic terms as a pulse moving to larger $|l|$ while losing energy, together with one moving to smaller $|l|$ while gaining energy. Figure 4 shows a somewhat exaggerated form of this rather striking signature.

At the initial instant, the energy gain by packet $A$ will exactly balance the loss 


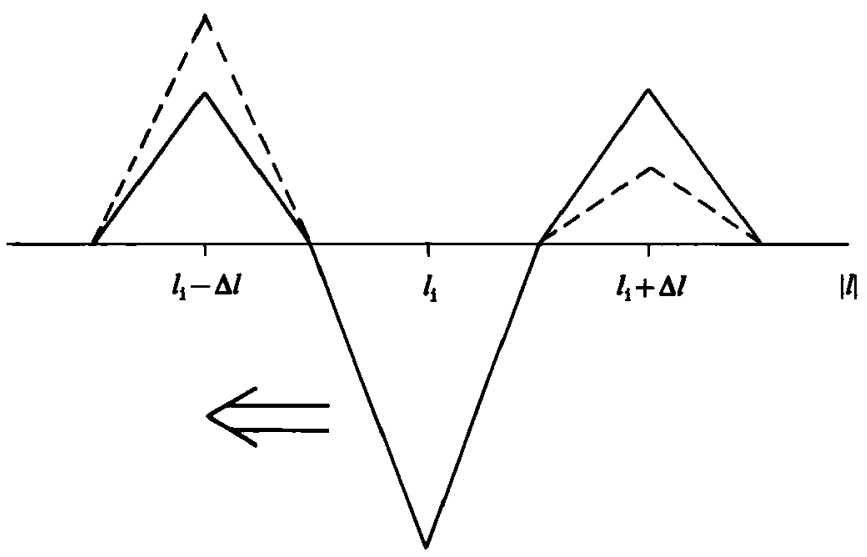

Figure 4. Schematic of enstrophy (-) and energy (---) transfer terms, scaled by $l_{1}^{2}$ to one another, for interaction between a disturbance of meridional scale $l_{1}$ and a zonal flow, assuming spectral symmetry. Arrow indicates the sense of the implied energy 'cascade'; the enstrophy loss at $l=l_{1}$ lies over the energy loss and so the latter is not visible.

by packet $B$. This is because $\mathrm{d} E / \mathrm{d} t=A \mathrm{~d} \hat{\omega} / \mathrm{d} t=A(\partial \hat{\omega} / \partial l) \mathrm{d} l / \mathrm{d} t$ will be equal and opposite for the two packets. As the pulses move apart, the changes in $l$ imply corresponding changes in $\partial \hat{\omega} / \partial l$ according to $\partial^{2} \hat{\omega} / \partial l^{2}=2 \beta_{0} k\left(k^{2}-3 l^{2}\right) /\left(k^{2}+l^{2}\right)^{3}$, using (3.4). It follows (noting that $A$ and $\mathrm{d} l / \mathrm{d} t$ are both negative in this case) that the energy gain by packet $A$ will exceed the energy loss by packet $B$ if $l^{2}>\frac{1}{3} k^{2}$, implying a net transfer of energy from the zonal flow to the disturbance; while if $l^{2}<\frac{1}{3} k^{2}$, then the net transfer will be in the opposite sense. This result is valid provided that the variation in $\mathbf{d} U / \mathrm{d} y$ is a higher-order effect, and not surprisingly it parallels the result for standing waves in plane Couette flow (e.g. Shepherd 1985, equation 7). The same result is also found in the case of a sinusoidal jet by using the exact conservation law discussed in $\$ 3.1$ : to leading order in $\gamma$ the net energy exchange vanishes, but the next-order effect is a net transfer which is to the disturbance if $l^{2}>\frac{1}{3} k^{2}$, and to the jet if $l^{2}<\frac{1}{3} k^{2}$ (Shepherd 1984, eq. 6.2).

If one now considers an ensemble of wave packets $(k, l)$ and $(k,-l)$ distributed homogeneously in space, rather than just a single pair, then this scenario is unchanged except that spectral dispersion about each composite 'pulse' enters immediately. The reason is that different pulses evolve at different rates $\mathrm{d} l / \mathrm{d} t$, since they operate under the influence of different basic-shear strengths. In the still more general case of a double ensemble, with homogeneous distributions of packets with different $k$ and $l$ but a single value of $\kappa$, one can easily see that the same picture will again emerge - as long as one is careful to look along 'cuts' defined by lines of constant $k$.

Although figure 4 depicts the initial spectral evolution of all disturbances that have spectral symmetry, it cannot hold indefinitely. To the up-scale pulse one of two things must happen: either it will reach $l=0$ and then turn back (viewed in terms of $|l|$ ), corresponding to a wave packet reflection off a turning line; or it will reverse direction before it reaches $l=0$, indicating that the packet has passed through the westerly jet maximum and that no turning lines exist. In the first case the pulse returns to its initial value of $|l|$, and is then identical with the initial down-scale pulse (neglecting any frictional loss); this is also true in the second case if the jet is symmetric about its westerly maximum.

As far as the down-scale pulse is concerned, there are again two possibilities: either 
the pulse will head off to very high $|l|$, corresponding to absorption near a critical line (anticipating the numerical experiments, we may presume the existence of high-order diffusion which would damp small-scale features); or it will reverse its tendency and move back up-scale, indicating that the packet has passed through the easterly jet maximum and that no critical lines exist. The first process is traditionally called 'absorption' because most of the wave energy is given up to the mean flow before the packet gets close to the critical line (e.g. Booker \& Bretherton 1967; Tung 1983): only the wave action (here just the wave enstrophy) is damped.

In summary, then, ray-tracing theory predicts the following outcome to the spectral transfer problem. Decomposing the disturbance wave field into wave packets, that part of the field represented by packets without critical lines (equivalently, that part projecting onto discrete rather than continuum modes; cf. Held 1985) will participate in no net wave-mean-flow interaction and can be ignored, the packets merely undergoing oscillations in $l$. The remainder of the disturbance field - which, for parameters relevant to this problem, represents by far the greatest part - will eventually be absorbed into the zonal flow: specifically, the disturbance enstrophy will be transferred to high $|l|$ and then damped by the presumed high-order diffusion, while in the process most of the wave energy will have been transferred back to the zonal flow. In the early stages of development, the wave energyespecially that associated with smaller zonal wavenumbers - can grow significantly at the expense of the zonal flow (the 'Orr effect'), at the same time becoming increasingly meridionally anisotropic $\left(v^{2}>u^{2}\right)$ at large scales, but this linear theory predicts such a process to be only temporary.

For completeness it may be noted that, in the case of linear Couette flow in an infinite domain, an initially isotropic disturbance field will maintain a constant energy level for all time (Shepherd 1985), implying no net energy exchange with the zonal flow. When the spatial spectrum is discrete, however, then asymptotic decay must eventually prevail; moreover spatial variations in the basic shear will also alter the result. But to the extent that the decaying packets balance the amplifying ones, the disturbance energy and enstrophy will both be approximately constant and the spectral transfer patterns, like figure 4, may temporarily look like the patterns of turbulent cascades in two-dimensional homogeneous turbulence, when analysed in terms of $\kappa$-although here the spectral dispersion takes place in $l$ for fixed $k$.

\subsection{Turbulent isotropization}

The ray-tracing theory of $\$ 3.2$ predicts strengthening of the zonal flow associated with irreversible, shear-induced transfer of disturbance enstrophy to large meridional wavenumber $l$. However, the problem under consideration is by assumption strongly nonlinear, and it is likely that nonlinear interactions will have a significant effect on the spectral transfers and on the net sense of the wave-mean-flow interaction. Here the hypothesis is put forward that the most important dynamical effect of turbulent interactions in this context will be their tendency to isotropize the transient eddy field by spreading the energy and enstrophy along curves of constant total wavenumber $\kappa$. Such an effect (which might in fact be considered as a phenomenological definition of turbulence in this problem) is completely inaccessible to the linear wave-mean-flow interaction, insofar as the latter can induce spectral transfer along, but not across, lines of constant zonal wavenumber $k$ (cf. §3.1).

The most thorough investigation of isotropization in two-dimensional turbulence seems to be that of Herring (1975), who considered homogeneous flow using a combination of closure methods and direct numerical simulation. Herring found that 
there is, indeed, a clear return to isotropy from anisotropic initial conditions, with an anisotropy decay rate proportional to the inverse of the 'eddy-turnaround time', $u_{0} / \lambda_{0}$ (for notation, see $\$ 2.2$ ). However, the process is much slower in two than in three dimensions, and is inherently non-local. The non-localness reflects the dominant process of the straining of small scales by very large ones, a characteristic feature of two-dimensional turbulence. It also leads to an effect whereby anisotropy initially concentrated at large scales spreads first to smaller scales, before eventually disappearing everywhere. Although Herring's study was for homogeneous turbulence only (a necessary restriction because of the closure methods used), one would expect it to have some relevance to the present context for scales much smaller than the scale of the inhomogeneity, provided that the relevant straining scales - not counting the basic flow, of course - were also locally homogeneous. The latter condition could well be quite stringent.

Herring (1975) interpreted the phenomenon of turbulent isotropization as a reflection of the known tendency of the inviscid spectrally truncated equations to seek (isotropic) statistical-mechanical equilibrium (Herring 1975, pp. 2254-5). There are, of course, many difficulties with this sort of argument, which need not be spelt out here (see e.g. Salmon 1982 and Shepherd 1987a). But the robust feature of the dynamical evolution is the increase of the flow entropy (Carnevale 1982), which manifests itself as spectral broadening. This broadening inevitably occurs along curves of constant $\kappa$ as well as across them. Numerical evidence (such as figure 10 of Haidvogel \& Held 1980) suggests that the former process tends to be more rapid than the latter, although it is hard to find a convincing explanation for this on statistical grounds.

If one now considers the combined picture, of shear-induced spectral transfer according to $\$ \$ 3.1$ and 3.2, together with turbulent isotropization as described here, it is evident that the simple picture of $\$ 3.2$ may be considerably altered. To the extent that nonlinear interactions destroy the phase tilt of the disturbances, the straining process will be disrupted: in particular, the strengthening of the zonal flow will be mitigated, as it relies on the maintenance of a phase tilt in the sense of packet $B$ of figure 3. Indeed, the possibility exists of a net transfer of energy to the disturbances, in complete contrast to the prediction of linear theory, to the extent that nonlinear effects preferentially disrupt the smaller-scale down-scale 'pulses' like packet $B$ in figure 3.

The crude scaling analysis of $\$ 2.2$ suggested that the relative importance for an eddy of nonlinear interactions with the basic flow and with other eddies is measured by the non-dimensional parameter $\alpha \equiv U_{0} / u_{0}$. Now that more insight has been gained into the dynamical characteristics of these processes, an improved estimate may be made. Taking Herring's result for the isotropization rate (the eddy-turnaround time may simply be viewed as a nonlinear 'scrambling' time), and the estimate (3.5) for the shear-induced spectral transfer, the two effects will be of comparable importance when

$$
\frac{\left(k^{2}+l^{2}\right) L_{0}}{k l U_{0}}=\frac{\lambda_{0}}{u_{0}} \Leftrightarrow \epsilon \equiv \frac{1}{2} \alpha \gamma \sin 2 \theta \equiv \frac{U_{0} \lambda_{0} \sin 2 \theta}{2 u_{0} L_{0}} \approx 1,
$$

where the wavevector angle $\theta \equiv \arctan (k / l)$ has been introduced. Equation (3.6) indicates that the relevant non-dimensional parameter is in fact $\epsilon$ rather than $\alpha$, and the former is much smaller than the latter. $\epsilon$ is obviously a sensitive function of $\theta$; for fixed $\alpha$ and $\gamma$ it is maximized, indicating the strongest basic-flow straining, when $k^{2}=l^{2}$. 


\section{Numerical experiments}

The theoretical discussion of the last section provides a qualitative description of the range of dynamical behaviour that might be expected in the present problem. However the theory fails to provide a complete solution to the full problem. One is therefore led to direct numerical simulation in order to understand the way in which the linear and nonlinear dynamics fit together, and in particular to determine the extent to which the dynamics can be considered as a competition between basic-flow straining and turbulent isotropization, as proposed in §3.3.

Three kinds of numerical simulation are considered. The first two kinds are 'spin-down' experiments: that is to say unforced, nearly inviscid evolution from spectrally localized initial conditions. A linear run, with wave-wave interactions suppressed, is shown first to exhibit the dynamics described in §3.2; then two fully nonlinear runs are described. The third kind of numerical simulation discussed is that of a forced-dissipative system run to statistical (turbulent) equilibrium. Such experiments are the closest analogues to geophysical systems that will be considered here, and they provide the most stringent test of the concepts advanced in §3.1.

One of the principal attractions of spin-down runs is that, because of their spectrally localized initial conditions and corresponding spectral gaps, the concept of a spectral cascade takes on a very concrete meaning which can be carefully observed in the time evolution of the experiment. This can give useful insight into the likely character of the nonlinear processes operating in spectrally broader, statistically steady, forced turbulence regimes, as has been amply demonstrated by the work of Rhines $(1975,1977)$.

\subsection{Description of the model}

The dynamical model to be considered is that of (2.1), or equivalently (2.3), taken over a square doubly periodic domain with a specific zonal basic flow. A form of $S$ in (2.1) that covers all the cases is given by

$$
\mathrm{S}(\psi)=-r \nabla^{2} \psi-\nu_{8} \nabla^{6} \psi+\nu_{8} \nabla^{8} \psi+F_{0}+F_{1},
$$

where the terms $F_{0}$ and $F_{1}$ will be explained presently. The choice of a doubly periodic domain rather than a zonal channel is made to ensure that inhomogeneities arise from the basic flow alone and not from the boundary conditions associated with channel walls. This study has already been restricted, on the basis of geophysical considerations, to a basic flow consisting of a large-scale zonal jet. The linear theory of $\$ 3.2$ suggests moreover that the basic flow ought to have variable shear in order to be considered representative, and that interesting phenomena may be associated with easterly and westerly flow maxima. A simple choice is that of a cosine jet; it is taken to have the gravest possible meridional structure in order to maximize the scale separation between the basic flow and the disturbance.

Without loss of generality, model units are chosen so that the domain has width $2 \pi$, and the disturbance has an r.m.s. velocity $u_{\text {rms }}=u_{0} \approx 1$. The other parameters are then determined on the basis of geophysical scalings, in $\$ 4.2$. The basic flow is chosen to take the form

$$
\Psi(y)=U_{\mathrm{b}} \sin y ; \quad U(y)=-U_{\mathrm{b}} \cos y \quad(0 \leqslant y<2 \pi) .
$$


The initial conditions for all the simulations are classical in this sort of study: namely a narrow isotropic ring, spectrally localized to

$$
\left.E_{\mathrm{D}}(k, l)\right|_{t=0}= \begin{cases}0.01 & \text { for } 9 \leqslant\left(k^{2}+l^{2}\right)^{\frac{1}{2}}<12, \\ 0 & \text { otherwise. }\end{cases}
$$

Phases are assigned randomly. The choice of the central wavenumber is meant to correspond roughly to the barotropic transient-energy input scale of the atmosphere, which is to say the first internal Rossby radius of deformation, but is not too critical for present purposes as long as it lies outside the cascade arrest scale $\kappa_{\beta}$ of Rhines (1975). $†$ The choice of initial conditions is of course important only for the spin-down experiments; the value of (4.3) in that context is that it highlights the spectral cascades of energy and enstrophy.

It has been found that, except in the later stages of linear simulations, a truncation wavenumber of $N=32$ (corresponding to a $64 \times 64$ set of grid points) is sufficient to handle the enstrophy cascade and to resolve the essential (large-scale) dynamics. This point was checked by monitoring the spectral transfers directly. The resolution is not high enough to give much of a hint of an enstrophy-cascading inertial subrange, but that is not the object of this study. For a more strongly inhomogeneous jet with smaller-scale structure, a higher resolution might well be required.

The numerical procedure employed is to solve the Fourier transform of either (2.1) or (2.3), with the appropriate $S$, advancing in time through a leapfrog algorithm with a leapfrog-trapezoidal step being taken every 23 time-steps to damp the computational mode. For these runs the time-step is $\Delta t=0.0015$. The nonlinear coupling arising from the advection terms is handled using the 'transform method', namely by performing the multiplication in physical space in a manner that conserves both energy and enstrophy, then transforming back to the spectral domain. Although the procedure is not alias-free, with high-order diffusion this should not present a problem since the aliasing is confined to high wavenumbers (Fox \& Orszag 1973). The evaluation of the derivatives is exact, as is the quadrature used for the Fourier transform.

For the spin-down runs, $r=0=\nu_{8}$ and $F_{1} \equiv 0$, and $\nu_{6} \neq 0$. In order for (4.2) to be an exact solution of (2.1), it is necessary to impose the extremely weak source-sink

$$
F_{0}=\nu_{6} \nabla^{6} \Psi=-\nu_{6} U_{\mathrm{b}} \sin y .
$$

In fact, for the spin-down runs performed the presence of $F_{0}$ had no discernible effect whatsoever. Note that the highly scale-selective biharmonic diffusion represents the only form of friction, so that the large-scale dynamics is nearly inviscid. It should be said that while the choice of a high-order diffusion operator is still a matter of some debate within the turbulence community (e.g. Basdevant \& Sadourny 1983; Sadourny \& Basdevant 1985), nevertheless for proper simulation of the large scales it seems necessary only to remove enstrophy at the smallest resolved scales - which all such operators do, in their own different ways, provided their coefficients are not too small (Bennett \& Haidvogel 1983).

For the forced-dissipative equilibrium runs, $v_{6}=0$ and $\nu_{8} \neq 0$ in (4.1). This implies the use of triharmonic rather than biharmonic diffusion, which confines the enstrophy dissipation even more to high wavenumbers (although the difference does not really matter so far as the large scales are concerned). The scale-independent

† On the other hand, by taking the central wavenumber as small as this the possibility of coherent-vortex formation is effectively precluded (McWilliams 1984). 
Ekman damping provided by $r$ is intended to enable a statistical equilibrium to be achieved. $F_{0}$ is again chosen to ensure that (4.2) is a solution of (2.1):

$$
F_{0}=r \nabla^{2} \Psi-\nu_{8} \nabla^{8} \Psi=-\left(r+\nu_{8}\right) U_{\mathrm{b}} \sin y .
$$

Equation (4.5) implies that whenever the sin $y$ component of the total stream function $\Psi+\phi$ falls below its initial (basic) amplitude, it will be forced; and whenever it rises above, it will be damped.

Finally, $F_{1}$ represents the intermediate-scale energy-enstrophy source, designed to simulate the injection due to baroclinic instability at roughly the scale of the Rossby deformation radius. Some such forcing is traditional in two-dimensional turbulence simulations (e.g. Basdevant et al. 1981), although its precise form is open to debate. Forcing of the 'negative viscosity' type (Basdevant et al. 1981) was tried first but was ultimately rejected, for two reasons: it was anisotropic in an uncontrollable sort of way, and it slowed the approach to statistical equilibrium. The simulations discussed here use instead

$$
F_{1}(\hat{\psi}(k, l))= \begin{cases}\mu \frac{\hat{\psi}(k, l)}{|\hat{\psi}(k, l)|^{2}} & \text { for } 9 \leqslant\left(k^{2}+l^{2}\right)^{\frac{1}{2}}<12 \\ 0 & \text { otherwise }\end{cases}
$$

which is easy to apply since (2.1) is solved spectrally. The attractive aspect of (4.6) is that it provides a constant energy/enstrophy input at each wavenumber in the forcing band and is thus both steady and isotropic: the injection due to $F_{1}$ alone is given by

$$
\frac{\mathrm{d}}{\mathrm{d} t} E(k, l)=\mu, \quad \frac{\mathrm{d}}{\mathrm{d} t} G(k, l)=\mu\left(k^{2}+l^{2}\right)
$$

While the enstrophy dissipation rate cannot be anticipated since it depends on the spectral distribution, the energy dissipation rate is effectively scale independent and can be anticipated, being dependent mainly on $r$ and hardly at all on $v_{8}$.

\subsection{Determination of parameter values}

To determine scales relevant to the atmosphere, summing up the stationary and transient components of energy from the FGGE-3A data (Boer \& Shepherd 1983) leads to

$$
\begin{aligned}
& E_{\text {stat }} \approx 100 \mathrm{~J} / \mathrm{kg} \Rightarrow U_{*} \approx 17 \mathrm{~m} / \mathrm{s}, \\
& E_{\text {trans }} \approx 50 \mathrm{~J} / \mathrm{kg} \Rightarrow u_{*} \approx 10 \mathrm{~m} / \mathrm{s},
\end{aligned}
$$

the asterisk subscript denoting dimensional values. Given $u_{\text {rms }} \approx 1$ in the model, this suggests choosing $U_{\mathrm{b}}=2 \sqrt{ } 2$ in (4.2) so that $\left\langle U^{2}(y)\right\rangle=4$ and thus the nondimensional parameter $\alpha$ of (2.8) takes the value of 2 . To find a lengthscale, choose the mid-latitude domain scale $L_{*}=24000 \mathrm{~km}$. Hence the model units are scaled by $\left(L_{1}, U_{1}, T_{1}\right)$, where

$$
\begin{gathered}
U_{1}=\frac{u_{*}}{1} \approx 10 \mathrm{~m} / \mathrm{s}, \quad L_{1}=\frac{L_{*}}{2 \pi} \approx 4 \times 10^{6} \mathrm{~m} \\
T_{1}=\frac{L_{1}}{U_{1}} \approx 4 \times 10^{5} \mathrm{~s} \approx 5 \text { days, } \beta_{0}=\frac{\beta_{0 *} L_{1}^{2}}{U_{1}} \approx 25 .
\end{gathered}
$$

With this scaling, the intermediate-scale forcing (4.6) is applied at lengthscales just smaller than the Rossby deformation radius of $1000 \mathrm{~km}$ (taking $10 \mathrm{~km}$ for the fluid, i.e. tropospheric, depth). 
To mimic the cascade of enstrophy to subgrid scales, $\nu_{6}$ or $\nu_{8}$ must be chosen so that the 'grid Reynolds number' $u_{\mathrm{rms}} / \nu_{6} N^{3}$ or $u_{\mathrm{rms}} / \nu_{8} N^{5}$ is less than about 10 (Bennett \& Haidvogel 1983). Here $N$ is the truncation wavenumber, where most of the dissipation should be concentrated. For $N=32$ and $u_{\mathrm{rms}}=1$, for example, this suggests the values $v_{6}=3 \times 10^{-6}$ or $v_{8}=3 \times 10^{-9}$, which are adopted here.

With regard to the forcing and Ekman dissipation in the equilibrium simulations, we can use Boer \& Shepherd's (1983) observational estimate of the maximum up-scale kinetic energy cascade rate, which is $4 \times 10^{-5} \mathrm{~J} /(\mathrm{kg} \mathrm{s})$. Assuming that this cascade originates from intermediate-scale baroclinic-to-barotropic conversion, one may take the same value as an estimate of the forcing parameter appropriate to a barotropic model; in fact it is probably a lower bound for the latter, but at least it gives an order-of-magnitude estimate. Putting this quantity in model units, using the scaling of (4.8), yields a total energy injection rate of 0.5 , which corresponds to $\mu=0.0025$ for each of the approximately 200 modes in the forced wavenumber band. Since the Ekman damping rate is given by $-2 r E$, this suggests taking $r=0.5$ if the 'damped' part of the spectrum (roughly $E-E_{0}$ ) is to maintain an energy level of 0.5 .

It is interesting that $r=0.5$ implies a dimensional spin-down timescale of five terrestrial days, which happens to be that which generally gives the best results for simple mid-latitude forced linear stationary-wave models (e.g. Held 1983). It is also consistent with conventional estimates of the frictional stress being roughly a fraction of a dyne $/ \mathrm{cm}^{2}$ (Jeffreys 1933).

\subsection{Linear spin-down: run A}

In this section the 'linear' dynamics, consisting of straining of the disturbance by the basic shear under the influence of $\beta$, is isolated by showing a spin-down experiment (run A) with wave-wave interactions suppressed. The experiment is performed by solving $(2.3)$ with the $J\left(\phi, \nabla^{2} \phi\right)$-term omitted, and with $S$ as described for spin-down runs in $\$ 4.1$.

Figure 5 shows the time evolution of the disturbance stream function and vorticity fields. For convenience, the profile of the basic flow (4.2) is superimposed on figure $5(b)$. The initial disturbance state (figures $5 a, b$ ) is homogeneous and isotropic, but by $t=1$ the vorticity shows the expected zonal anisotropy, $u^{2}>v^{2}$ (figure 5d). However the stream function for the same time exhibits meridional anisotropy, $v^{2}>u^{2}$ (figure $5 c$ )! This provides a vivid reminder that it is the disturbance vorticity, and not the stream function, that is advected (and strained) by the basic flow. Inasmuch as the stream function and the vorticity can be interpreted respectively as low-pass and high-pass spatial filters of the disturbance velocity field (cf. Rhines $1977, \S 3$ ), figure $5(c)$ picks out those wave components that have been amplified according to the 'Orr effect'; evidently some $k=4$ components are locally at the stage of maximum amplification corresponding to figure $2(b)$. By $t=4$ (figures $5 e, f$ ) the disturbance has been nearly eliminated, except in the regions of weak basic shear. Note that the meridional scales of $\phi$ are largest in the westerly jet, in agreement with ray-tracing theory. However, by this time the resolution is becoming inadequate to describe the fine details of the linear evolution.

Figure 6 shows a time sequence of time-averaged conversion terms for disturbance energy and enstrophy, $C_{\mathrm{D}}(k=4 ; l)$ and $\kappa^{2} C_{\mathrm{D}}(k=4 ; l)$, considered as functions of $l$ for fixed $k=4$. For the purpose of clarity, the figure shows the development of only a part of run $A$, namely the flow that develops from modes $(k, l)=(4, \pm 9)$ at $t=0$. (Since run A is linear, its development is a superposition of the development of the individual modes.) Shear-induced, spectral transfer of disturbance enstrophy along 
(a)



(c)

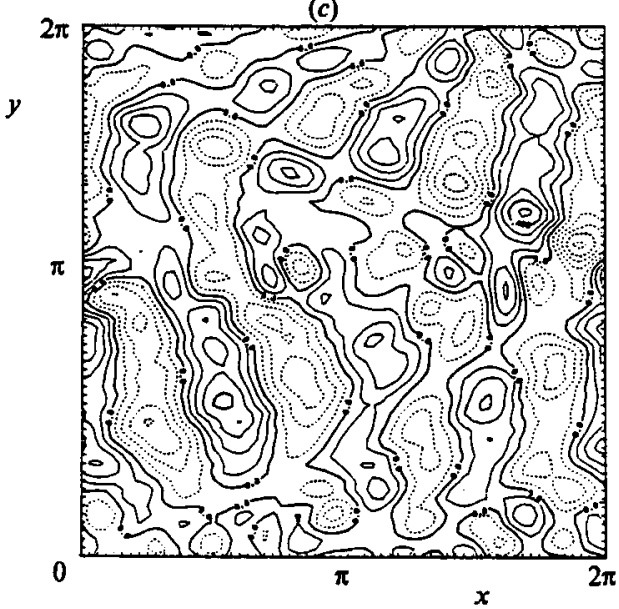

(e)

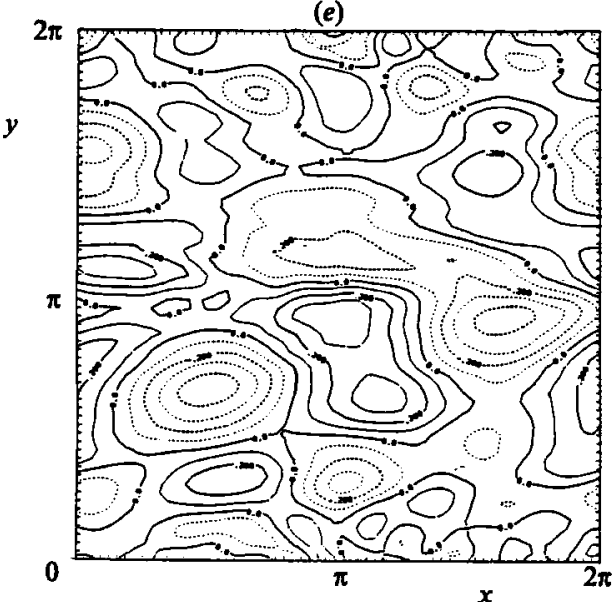

(b)

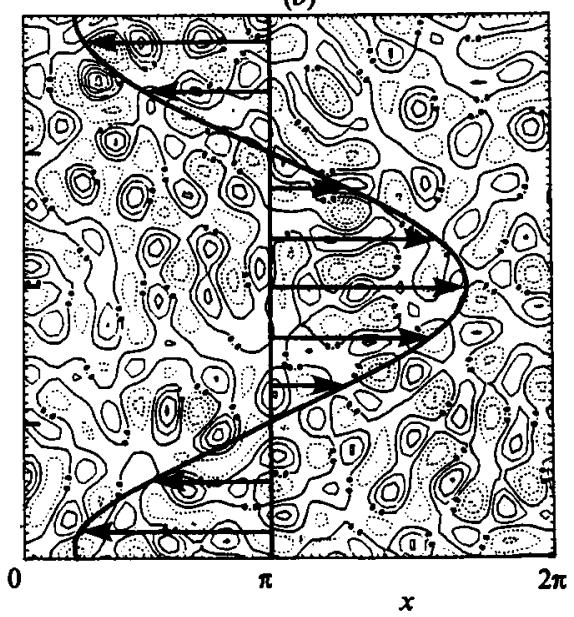

(d)

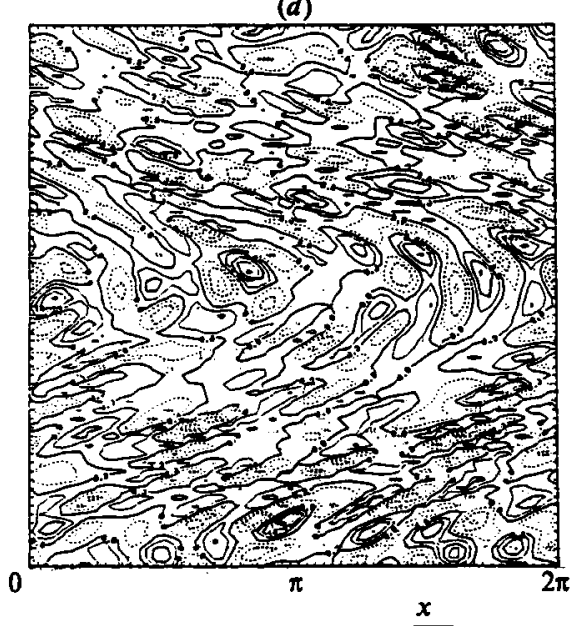

(f)

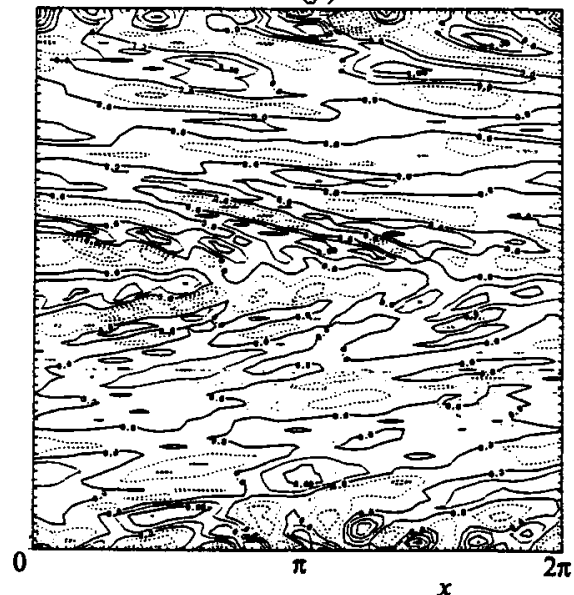

Figure 5. Instantaneous spatial maps of the disturbance stream function and vorticity fields $\phi(x, y)$ and $\nabla^{2} \phi(x, y)$ for linear spin-down run A. Solid lines denote positive values, dashed lines negative. (a) $\phi, t=0 ;(b) \nabla^{2} \phi, t=0 ;(c) \phi, t=1 ;(d) \nabla^{2} \phi, t=1 ;(e) \phi, t=4 ;(f) \nabla^{2} \phi, t=4$. The profile of the basic flow is superimposed on $(b)$. 

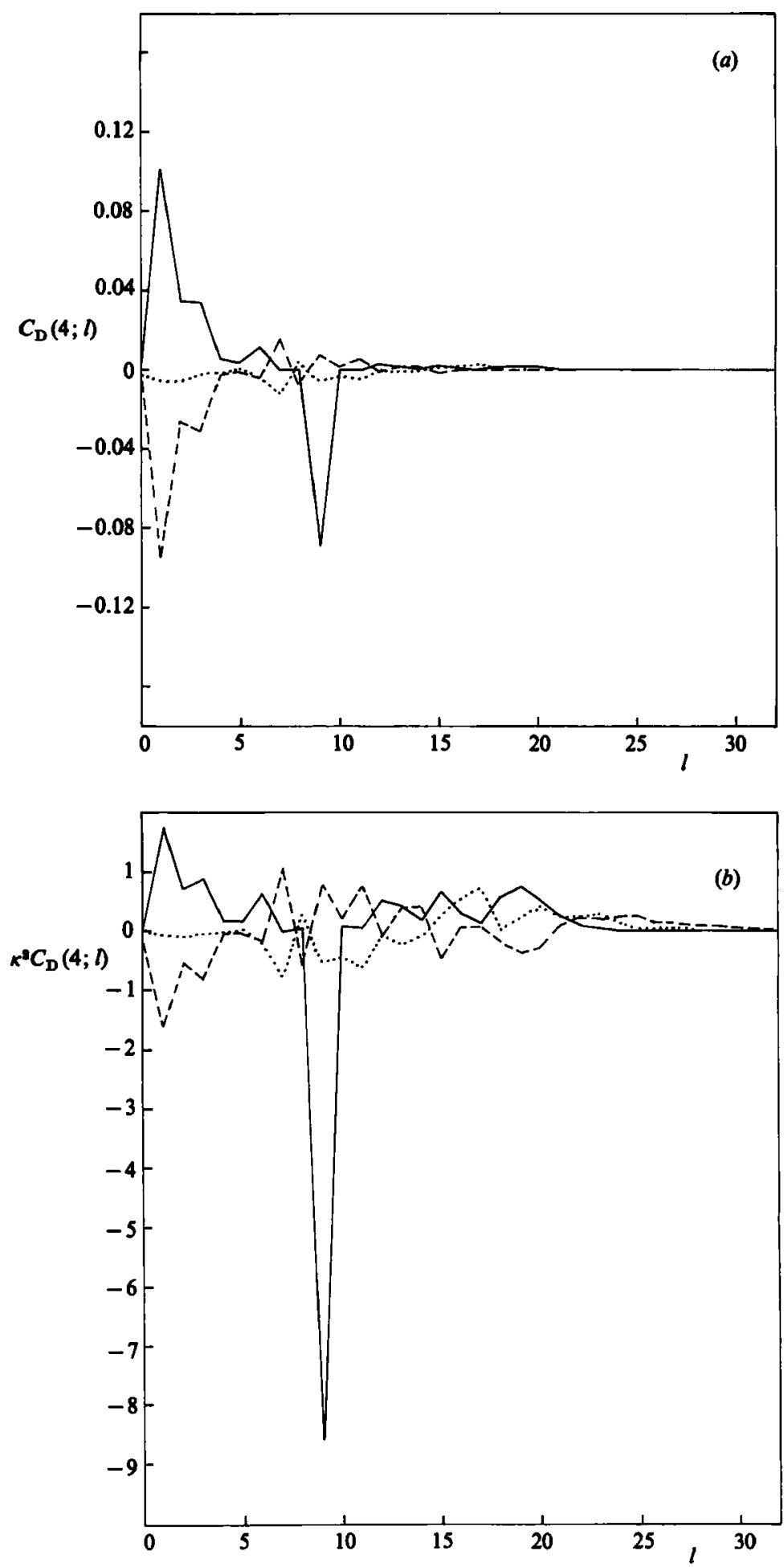

Fraure 6. (a) Energy conversion term $C_{\mathrm{D}}(k ; l)$ and $(b)$ enstrophy conversion term $\kappa^{2} C_{\mathrm{D}}(k ; l)$ for fixed $k=4$ as a function of $l$, averaged in time, for that part of the linear spin-down run $A$ originating from modes $(k, l)=(4, \pm 9): t=0-1(-), t=1-2(---), t=2-3(\cdots \cdots)$. 
lines of constant zonal wavenumber $k$, anticipated by the arguments of $\$ \$ 3.1$ and 3.2 , is clearly evident. The enstrophy (figure $6 b$ ) is very nearly conserved along the line $k=4$ in the time evolution - the sum of the terms over all $l$ is less than $5 \%$ of the largest term - but the energy (figure $6 a$ ) is not at all conserved. The development during $t=0-1$ (the solid line of the figures) is very like the schematic of figure 4, except that reflection of the 'up-scale pulse' of disturbance enstrophy at $l=0$ is already taking place. The Orr effect of up-scale transfer and energetic amplification shows up strongly in the energy terms for $t=0-1$, as expected from figure $5(c)$, but is almost completely reversed by $t=2$. Figure 6 demonstrates clearly the reversible nature of the Orr effect when nonlinear effects are ignored.

\subsection{Nonlinear spin-down: runs $\mathrm{B}$ and $\mathrm{C}$}

To try to understand the combined effects of the linear and the nonlinear processes, two more spin-down simulations are shown. Run B is classical beta-plane turbulence, namely (2.1) with $U_{\mathrm{b}}=0$ (no basic flow). Run $\mathrm{C}$ has all processes included.

Snapshots of the disturbance stream function and vorticity fields at $t=4$ are shown in figures 7 and 8 , and are to be compared with figures $5(e, f)$. Qualitatively one sees that run $\mathrm{C}$ is quite distinct from the other two. While run $\mathrm{B}$ exhibits the development of zonally elongated eddies of meridional scale $\kappa_{\beta}^{-1}$ described by Rhines (1975) and Holloway \& Hendershott (1977) (figure 7a), together with the small-scale spatial homogeneity and 'teasing out' of vortices associated with pure two-dimensional turbulence (figure $7 b$ ), run $\mathrm{C}$ has significantly excited the largest scales of motion (figure 8a)-thus penetrating the 'Rhines radius' $\kappa=\kappa_{\beta}$-and shows marked shear-induced anisotropy in the vorticity field (figure $8 b$ ). But run $\mathrm{C}$ also contrasts sharply with the linear run $A$, as the former maintains much greater spatial homogeneity in the disturbance field than does the latter, and also appears to trap a significant amount of energy at the largest scales.

These features are quantified in a time series of time-averaged spectra for all three runs, shown in figures 9,10 and 11. The spectra show the zonal and meridional components of disturbance energy, defined in (2.12), as functions of the total wavenumber $\kappa$. (In all spectral data presented as a function of $\kappa$, contributions from modes $\kappa$ with $n \leqslant \kappa<n+1, n$ being an integer, are assigned to wavenumber $\kappa=n$.) Run A (figure 9) initially exhibits large-scale meridional and small-scale zonal anisotropy (i.e. $v^{2}>u^{2}$ and $u^{2}>v^{2}$ respectively), a result of the shear-induced spectral transfer - entirely a linear process. However, by $t=9-10$ (figure 9d) most of the energy has evidently been lost in the interaction with the basic flow, as predicted in \$3.2. The classical up-scale energy cascade associated with twodimensional beta-plane turbulence, manifested in run B (figure 10), differs from this linear shear-induced transfer in at least four important respects: it is slower; it is fairly isotropic; it is arrested at the Rhines wavenumber $\kappa_{\beta}$, which is roughly $\kappa_{\beta} \approx 5$ here; and, finally, it is irreversible.

Turning now to run $\mathrm{C}$ (figure 11), the rapidity of the initial up-scale cascade and the associated large-scale meridional anisotropy (figure $11 a$ ) suggest the dominant role of the shear-induced transfer mechanism in this range. But the small scales are relatively isotropic during $t=0-2$, a feature that is inexplicable by linear theory and must be attributed to nonlinear effects. In contrast with run $B$, there is no up-scale cascade arrest at $\kappa_{\beta}$; but in contrast with run $A$, the up-scale transfer is irreversible (figure $11 d$ ). The degree of isotropy at the largest scales during $t=1-3$ suggests that isotropization by nonlinear processes is indeed the mechanism that makes the linear up-scale transfer irreversible. An examination of two-dimensional $(k, l)$ spectra (not 
(a)



(b)

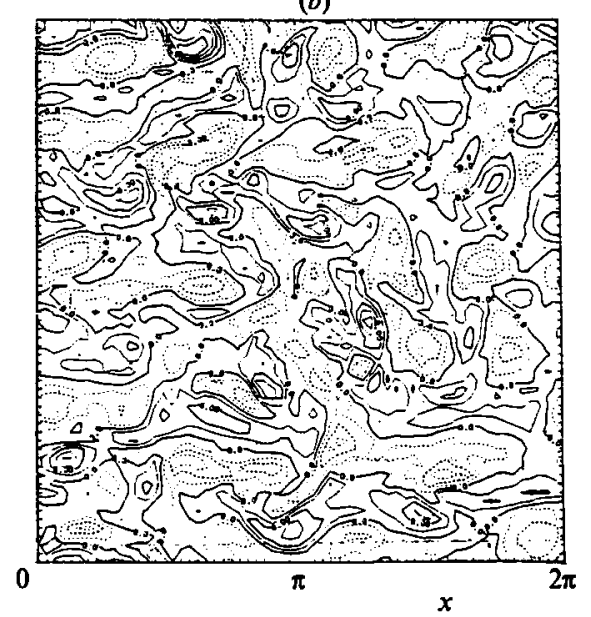

Froure 7. Instantaneous spatial maps at $t=4$, nonlinear spin-down run B (no basic flow), of $(a)$ stream function and $(b)$ vorticity.

(a)

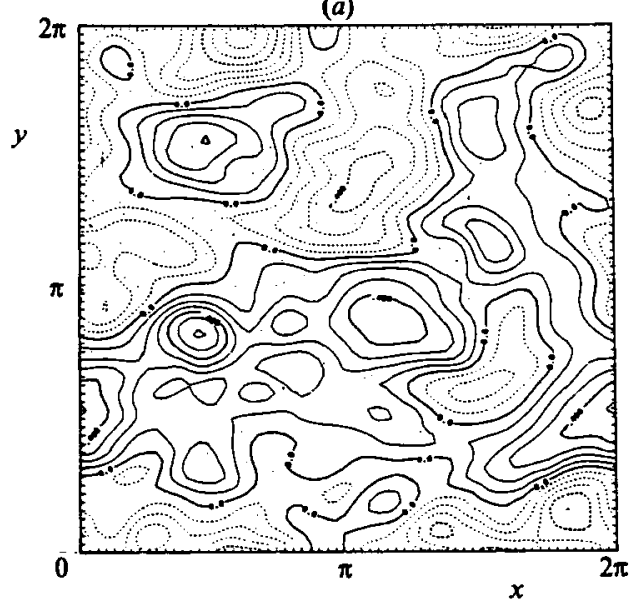

(b)

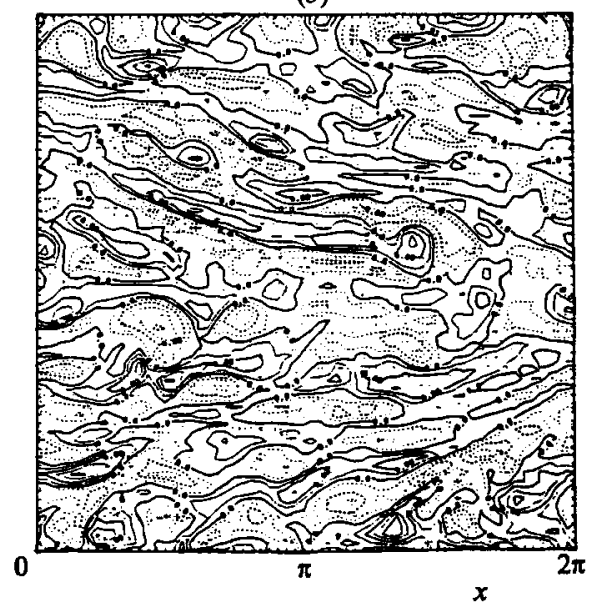

FIGURe 8. Instantaneous spatial maps at $t=4$, nonlinear spin-down run $C$ (with basic flow), of (a) disturbance stream function and $(b)$ disturbance vorticity.

shown) confirms that the spectra are indeed approximately isotropic in the sense that $E_{\mathrm{D}}(\kappa)$ tends to be a function of $\kappa$ alone; the key point is that, as anticipated by the arguments of $\S 3.3$, the anisotropic tendency of the shear-induced transfer is smoothed towards isotropy.

The nature of the shear-induced transfer is evident from figure 12, which exhibits the time evolution of $C_{\mathrm{D}}(k=4 ; l)$ and $\kappa^{2} C_{\mathrm{D}}(k=4 ; l)$ and which may be compared with figure 6. The qualitative comments made there apply here, but the initial up-scale transfer is less cleanly reversed here compared with the linear run.

\subsection{Spectral diagnostic formulae (continued)}

To this point in the paper, the problem under consideration has been studied in the context of a (nonlinear) disturbance to a specified zonal basic flow. But in many cases, particularly for a flow in forced-dissipative equilibrium, it is more appropriate to 


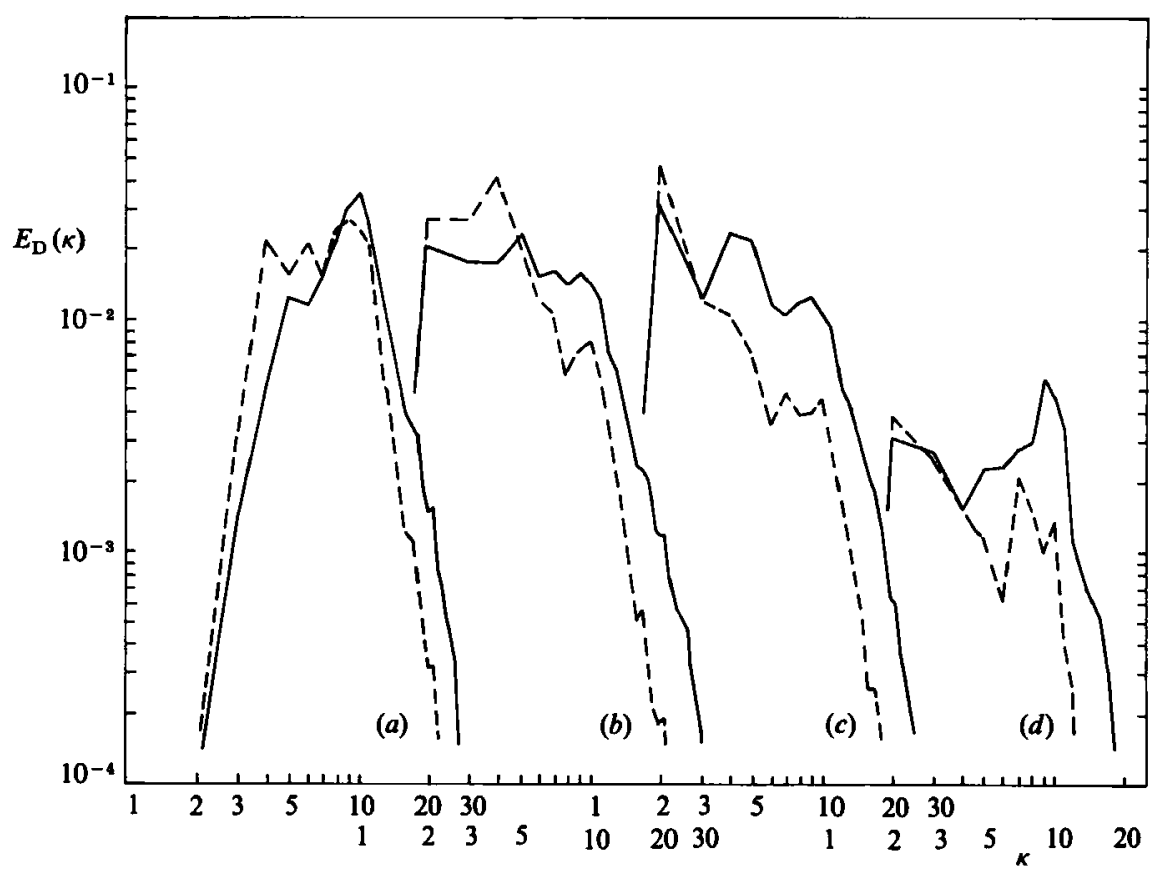

Fiavre 9. Disturbance energy spectrum $E_{\mathrm{D}}(\kappa)$ for run $A$, averaged in time, and decomposed into zonal $(\longrightarrow)$ and meridional (---) components. $(a) t=0-1 ;(b) t=1-2 ;(c) t=2-3 ;(d) t=9-10$.

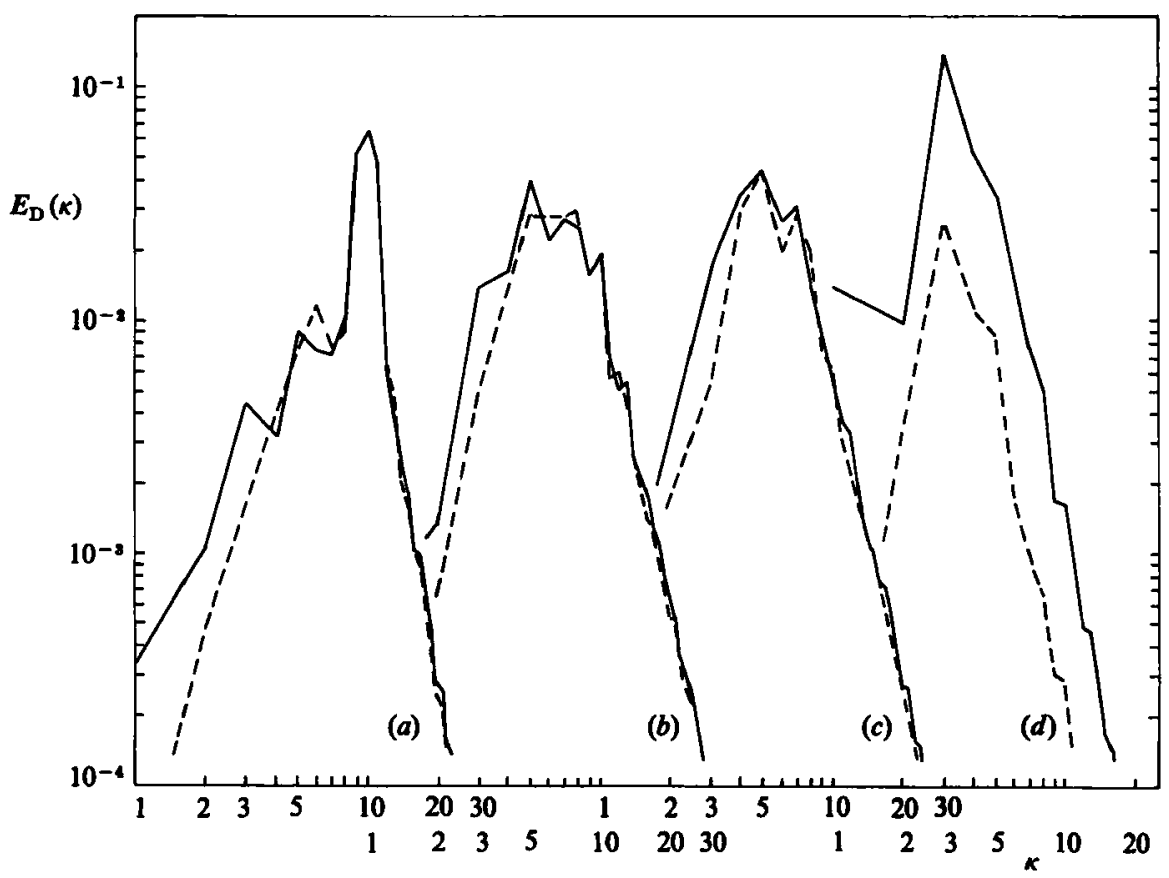

Figure 10. As in figure 9, but with $E(\kappa)$ (energy of the total flow, equal to $E_{\mathrm{D}}(\kappa)$ since $U_{\mathrm{b}}=0$ ) for run $B$. The Rhines cascade-arrest wavenumber $\kappa_{\beta}$ is about 5 . 


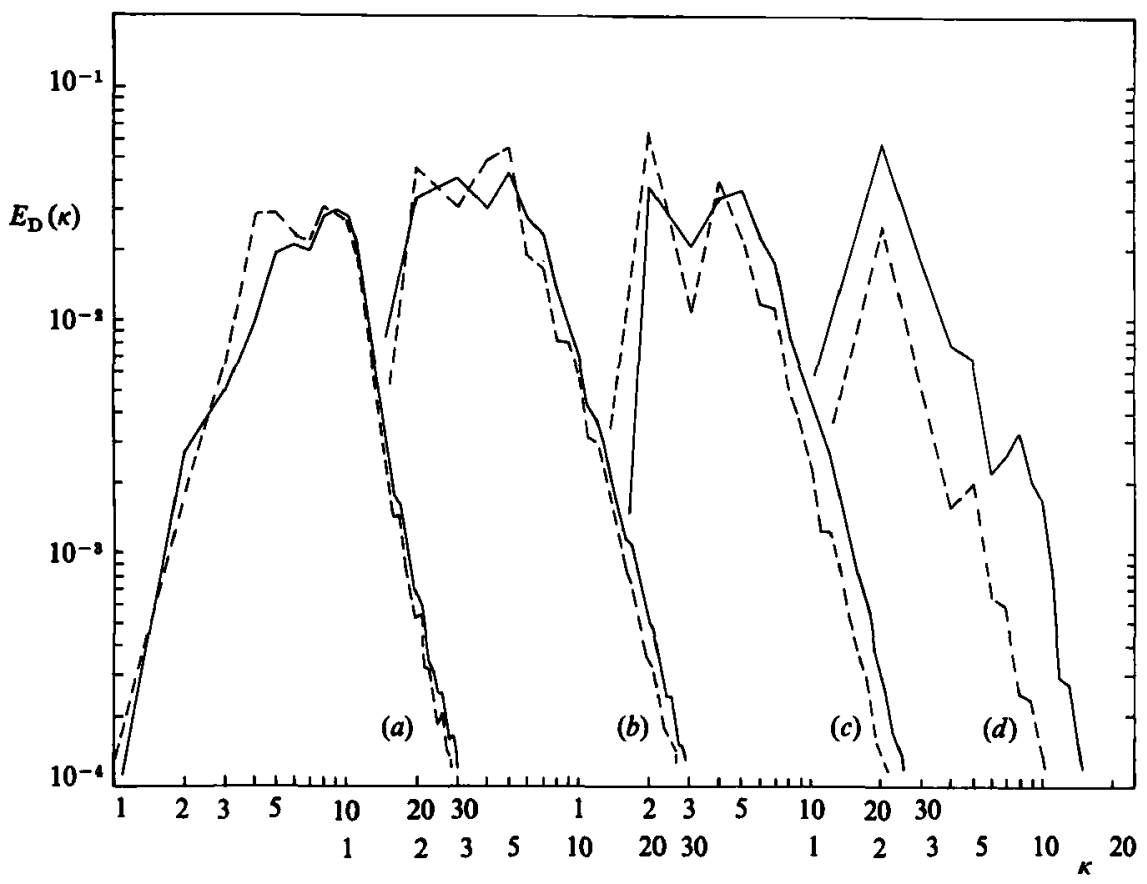

Figure 11. As in figure 9, but with $E_{\mathrm{D}}(\kappa)$ for run C.

examine the stationary and transient budgets instead. (As discussed in \$3.1, the essential theoretical arguments of this paper apply equally for both approaches.) Rather than (2.3), one must consider the pair of equations

$$
\begin{gathered}
\mathrm{J}\left(\bar{\psi}, \nabla^{2} \bar{\psi}\right)+\beta_{0} \bar{\psi}_{x}=\overline{\mathrm{S}}(\psi)-\overline{\mathrm{J}\left(\psi^{\prime}, \nabla^{2} \psi^{\prime}\right)} \\
\nabla^{2} \psi_{t}^{\prime}+\mathrm{J}\left(\psi^{\prime}, \nabla^{2} \psi^{\prime}\right)+\mathrm{J}\left(\bar{\psi}, \nabla^{2} \psi^{\prime}\right)+\mathrm{J}\left(\psi^{\prime}, \nabla^{2} \bar{\psi}\right)+\beta_{0} \psi_{x}^{\prime}=\mathrm{S}^{\prime}(\psi)+\overline{\mathrm{J}\left(\psi^{\prime}, \nabla^{2} \psi^{\prime}\right),}
\end{gathered}
$$

where $\psi \equiv \bar{\psi}+\psi^{\prime}$, the overbar denoting a time average over some interval. The transient budgets are obtained by multiplying the Fourier transform of $(4.9 b)$ by $\psi^{\prime *}(\kappa)$ or by $-\kappa^{2} \psi^{\prime *}(\kappa)$, adding the c.c., and then taking a time average; the result is completely analogous to (2.13) and (2.14), namely

$$
\begin{gathered}
\frac{\partial}{\partial t} E_{\mathrm{T}}(\kappa)=0=I_{\mathrm{T}}(\kappa)+C_{\mathrm{T}}(\kappa)+D_{\mathrm{T}}(\kappa), \\
\frac{\partial}{\partial t} G_{\mathrm{T}}(\kappa)=0=\kappa^{2} I_{\mathrm{T}}(\kappa)+\kappa^{2} C_{\mathrm{T}}(\kappa)+\kappa^{2} D_{\mathrm{T}}(\kappa),
\end{gathered}
$$

where the terms are defined in the Appendix and are exactly as for the disturbance quantities, with $\psi^{\prime}$ replacing $\phi, \bar{\psi}$ replacing $\Psi$, and a time average being taken of the whole quantity.

However the stationary flow does not satisfy the governing equation (2.1), but rather the balance equation $(4.9 a)$. To obtain stationary budgets, multiply the Fourier transform of $(4.9 a)$ by $\hat{\psi}^{*}(\kappa)$ or by $-\kappa^{2} \overline{\hat{\psi}}^{*}(\kappa)$ and add the c.c.; this yields

$$
\begin{aligned}
\frac{\partial}{\partial t} E_{\mathrm{S}}(\kappa) & =0=I_{\mathrm{S}}(\boldsymbol{\kappa})+C_{\mathrm{S}}(\boldsymbol{\kappa})+D_{\mathrm{S}}(\boldsymbol{\kappa}), \\
\frac{\partial}{\partial t} G_{\mathrm{S}}(\boldsymbol{\kappa})=0 & =\kappa^{2} I_{\mathrm{S}}(\boldsymbol{\kappa})+\kappa^{2} C_{\mathrm{S}}(\boldsymbol{\kappa})+\kappa^{2} D_{\mathrm{S}}(\boldsymbol{\kappa}) .
\end{aligned}
$$



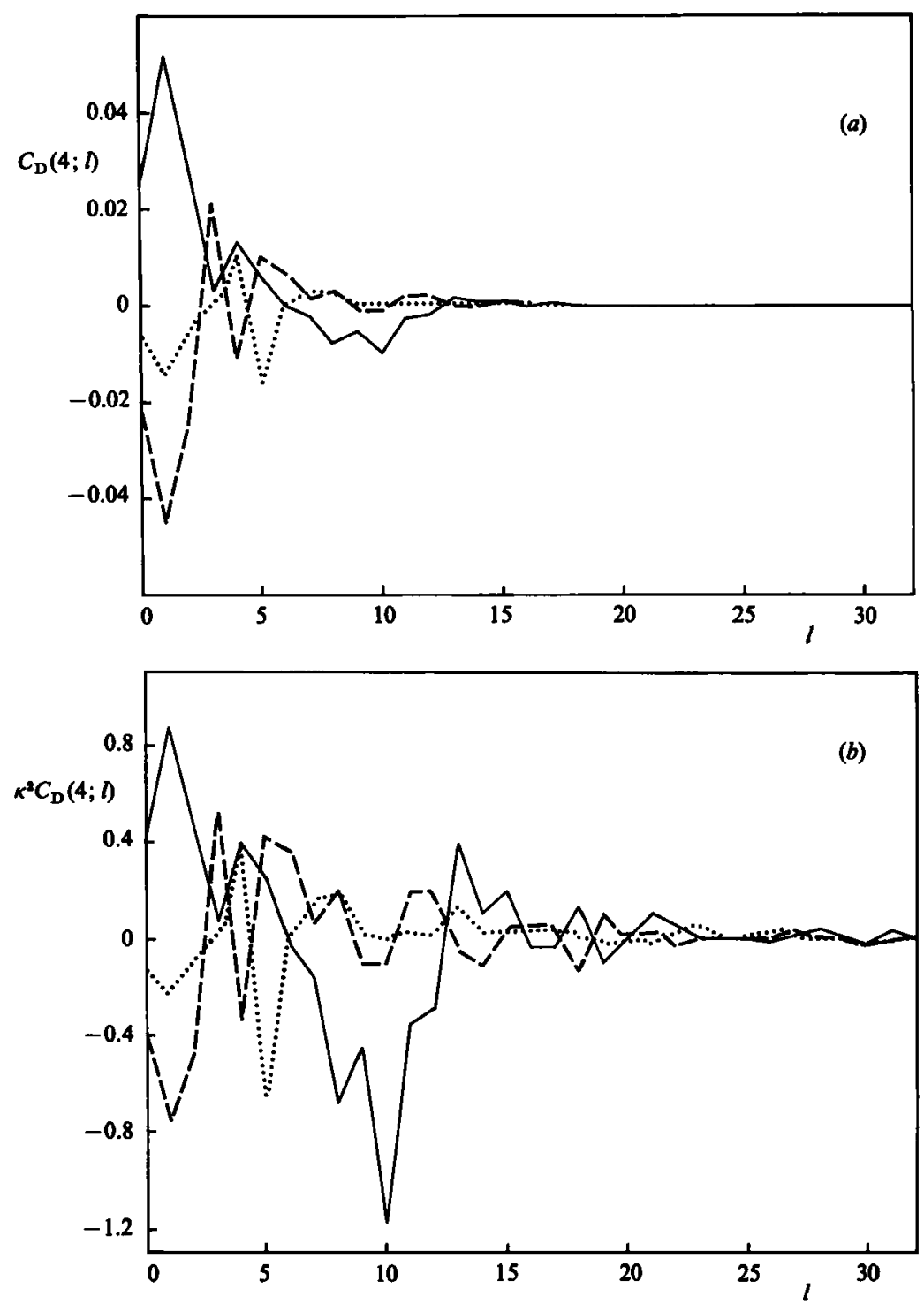

Fiadre 12. (a) Energy conversion term $C_{\mathrm{D}}(k ; l)$ and $(b)$ enstrophy conversion term $\kappa^{2} C_{\mathrm{D}}(k ; l)$ for fixed $k=4$ as a function of $l$, averaged in time, for run $\mathrm{C}: t=0-1(-), t=1-2(---)$, $t=2-3(\cdots \cdots)$.

Again the terms are defined in the Appendix. The stationary energy $E_{\mathrm{S}}(\kappa)$ is given by (2.11) with $\bar{\psi}$ replacing $\phi . I_{\mathrm{S}}(\boldsymbol{\kappa})$ represents the energy transfer to $\boldsymbol{\kappa}$ arising from interactions involving only stationary components, $C_{\mathrm{s}}(\boldsymbol{\kappa})$ that arising from mixed stationary-transient interactions; the two terms are respectively associated with the quantities $\mathrm{J}\left(\bar{\psi}, \nabla^{2} \bar{\psi}\right)$ and $\bar{J}\left(\psi^{\prime}, \nabla^{2} \psi^{\prime}\right)$ in $(4.9 a)$.

It is easily seen that $I_{\mathrm{T}}(\boldsymbol{\kappa})$ and $I_{\mathrm{S}}(\boldsymbol{\kappa})$ represent true interaction terms for both energy and enstrophy, the first effecting transfers between transient waves, the second between stationary waves; thus

$$
\sum_{\kappa} I_{\mathrm{T}}(\boldsymbol{\kappa})=\sum_{\kappa} \kappa^{2} I_{\mathrm{T}}(\kappa)=\sum_{\kappa} I_{\mathrm{S}}(\kappa)=\sum_{\kappa} \kappa^{2} I_{\mathrm{S}}(\kappa)=0
$$


Note that the stationary-transient conversion terms seen by the stationary flow, $C_{\mathrm{S}}(\kappa)$ and $\kappa^{2} C_{\mathrm{s}}(\kappa)$, are not the same at each wavenumber as the conversion terms seen by the transient flow, $C_{\mathrm{T}}(\kappa)$ and $\kappa^{2} C_{\mathrm{T}}(\kappa)$. However, they are consistent in an integrated sense:

$$
\sum_{\kappa} C_{\mathrm{S}}(\boldsymbol{\kappa})=-\sum_{\kappa} C_{\mathrm{T}}(\boldsymbol{\kappa}) ; \quad \sum_{\boldsymbol{\kappa}} \kappa^{2} C_{\mathrm{S}}(\boldsymbol{\kappa})=-\sum_{\boldsymbol{\kappa}} \kappa^{2} C_{\mathrm{T}}(\boldsymbol{\kappa})
$$

Equation (4.13) may be verified in physical space using integration by parts, or in the following direct fashion. Define

$$
I_{\mathrm{ST}}(\boldsymbol{\kappa}) \equiv C_{\mathrm{S}}(\boldsymbol{\kappa})+C_{\mathrm{T}}(\boldsymbol{\kappa})=\bar{I}(\boldsymbol{\kappa})-I_{\mathrm{S}}(\boldsymbol{\kappa})-I_{\mathrm{T}}(\boldsymbol{\kappa}),
$$

where $I(\kappa)$ is the interaction term computed for the full stream function $\psi$. But the right-hand side of (4.14) consists entirely of interaction terms, which vanish when integrated as in (4.12); hence

$$
\sum_{\kappa} I_{\mathrm{ST}}(\kappa)=0 \text { and similarly } \sum_{\kappa} \kappa^{2} I_{\mathrm{ST}}(\kappa)=0,
$$

implying (4.13). One may reconcile (4.10) and (4.11) at each $\kappa$ by rewriting $(4.10 a)$ as

$$
I_{\mathrm{T}}(\boldsymbol{\kappa})+I_{\mathrm{ST}}(\kappa)-C_{\mathrm{S}}(\kappa)+D_{\mathrm{T}}(\boldsymbol{\kappa})=0,
$$

with $I_{\mathrm{ST}}(\kappa)$ representing an interaction between transient waves induced by the catalytic though energetically passive presence of the stationary flow. The physical content of such a term must be considered carefully, however, as the definition is not free from ambiguity; all that has been done here is that a particular partition of $C_{\mathrm{T}}(\boldsymbol{\kappa})$ into interaction and conversion effects has been chosen. Whether the partition is physically meaningful is a question that can only be addressed within the context of a specific problem. In fact, for the present case of a scale separation between the stationary and transient flow components, the net enstrophy conversion is negligible (i.e. both sides of $(4.13 b)$ vanish to leading order) and so $\kappa^{2} C_{\mathrm{T}}(\kappa)$ can be mainly regarded as an interaction term.

\subsection{Nonlinear spin-down: run $\mathrm{C}$ (continued)}

The diagnostics introduced in the previous section are now used to examine the average dynamical behaviour over the active part of run C. Figure 13 shows the stationary-transient energy conversion terms, $C_{\mathrm{S}}(\kappa)$ and $C_{\mathrm{T}}(\kappa)$, as well as the corresponding terms for enstrophy. The time interval involved is $t=0-15$, beyond which the transfers are very weak. Since, by (4.13), the sums of the conversion terms must cancel, the smallness of $\kappa^{2} C_{\mathrm{S}}(\kappa)$ implies that $\kappa^{2} C_{\mathrm{T}}(\kappa)$ may be considered as a true interaction term for the transient flow, with the (time-) mean flow acting purely as a catalyst. But the net induced transfer of transient enstrophy is down-scale, or to higher $l$, and this leads to a net energetic forcing of the stationary flow. Two features are especially noteworthy about the induced transfer seen in figure $13(b)$. The first is that the initial down-scale transfer was quickly arrested by nonlinear effects, with very little transfer past $\kappa=15$. However the enstrophy dissipation, also shown in figure 13(b), implies that there was significant transfer to the smallest scales of motion, and this must therefore have been carried by the turbulent rather than the stationary-transient interactions. Such a result is not so surprising when one considers that for these scales, the non-dimensional parameter $\epsilon$ of $(\mathbf{3 . 6})$ is less than 0.1 .

The second point to note here is that there is also transfer from the vicinity of the cascade-arrest wavenumber, $\kappa=4,5$, and the implied source of transient enstrophy 

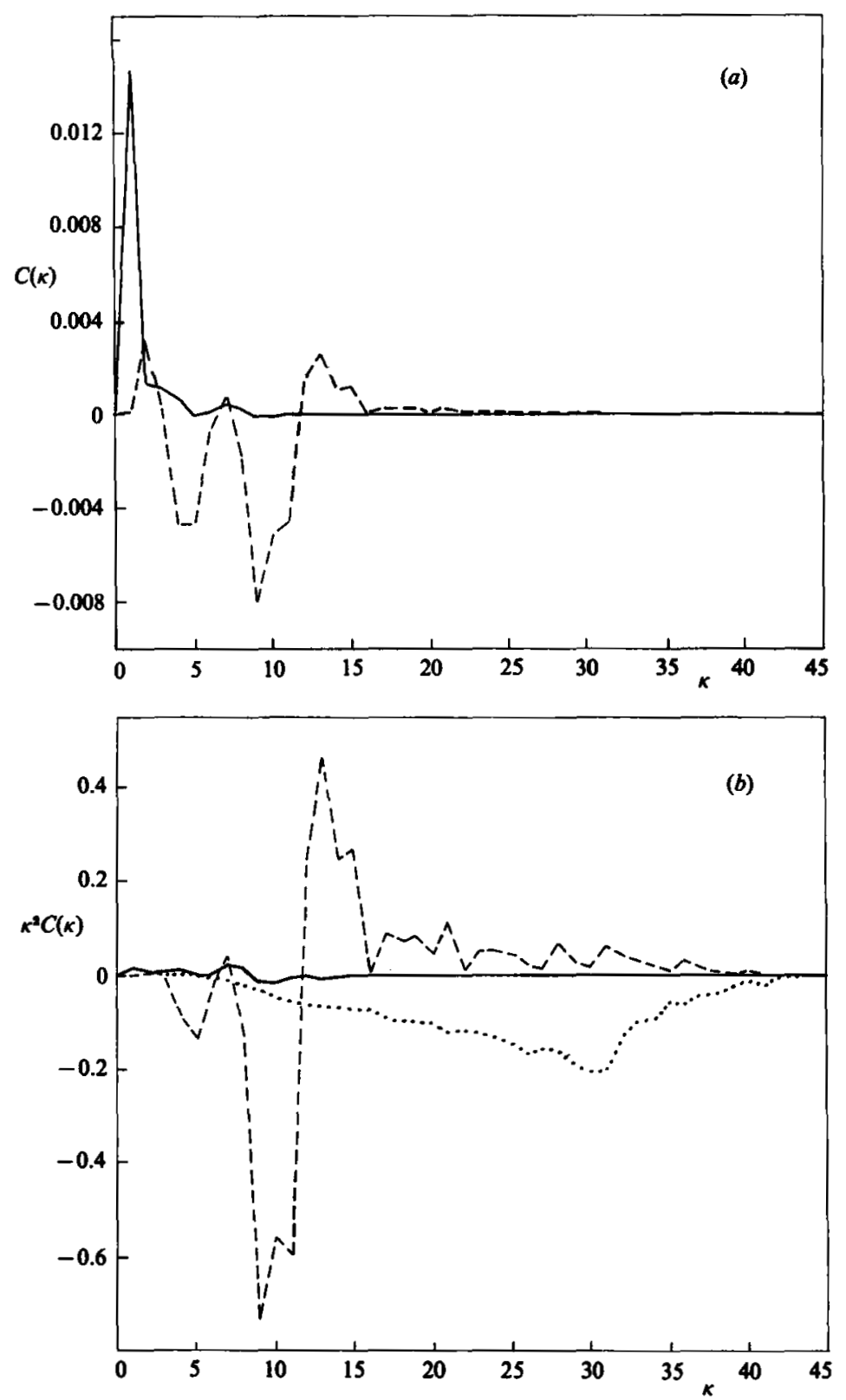

Frave 13. (a) Energy conversion terms $C_{\mathrm{S}}(\kappa)(-)$ and $C_{\mathrm{T}}(\kappa)(----)$, and $(b)$ enstrophy conversion terms $\kappa^{2} C_{\mathrm{S}}(\kappa)(-)$ and $\kappa^{2} C_{\mathrm{T}}(\kappa)(---)$, for run $\mathrm{C}$, averaged over $t=0-15$. In $(b)$ the dotted line shows the time-averaged dissipation of enstrophy, which outside of the initial scales $9 \leqslant \kappa<12$ must be balanced by nonlinear transfer less net accumulation.

at these scales can only be an up-scale turbulent cascade from the initial scales. This conclusion is confirmed by figure 14, which demonstrates the relative role of the 'induced' and 'turbulent' spectral transfers of energy in run C. Specifically, figure 14 shows the energy fluxes, which are defined by

$$
F_{\mathrm{ST}}(\kappa)=-\sum_{n=0}^{\kappa} I_{\mathrm{ST}}(n), \quad F_{\mathrm{T}}(\kappa)=-\sum_{n=0}^{\kappa} I_{\mathrm{T}}(n)
$$




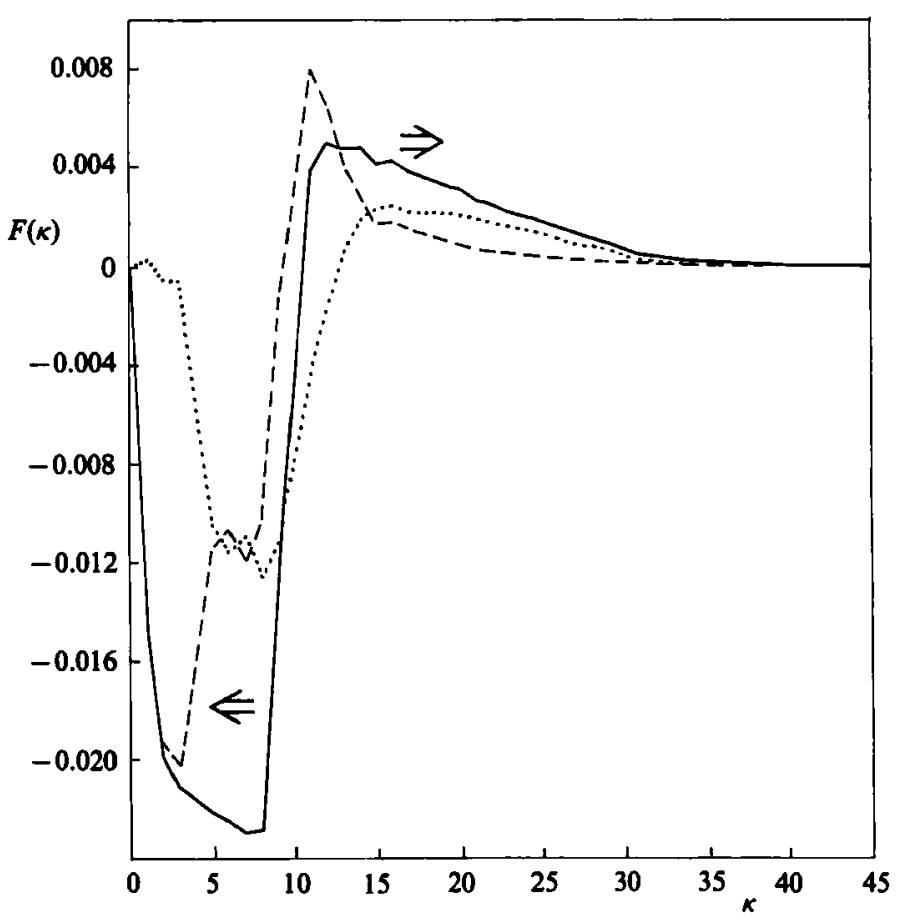

FIGURE 14. Nonlinear spectral fluxes of energy, run C, averaged over $t=0-15: F_{\mathrm{ST}}(\kappa)(----)$; $F_{\mathrm{T}}(\kappa)(\cdots \cdots)$; and $F(\kappa)(-)$, the latter representing the nonlinear interactions computed for the total stream function $\psi$. The residual, $F-F_{\mathrm{ST}}-F_{\mathrm{T}}=F_{\mathrm{S}} \equiv-\Sigma I_{\mathrm{S}}$, is effectively zero. Arrows denote the implied sense of the flux in the spectral domain.

respectively. A positive value of the flux denotes net transfer to smaller scales, that is to say down-scale transfer. Apparently there is a classical up-scale energy flux, as in two-dimensional beta-plane turbulence (also run B), which is arrested at $\kappa_{\beta}$. But the disturbance can be carried through $\kappa_{\beta}$, up to the largest scales of motion, by the induced transfer mechanism, and this same mechanism also acts around the input scales. Once the spectrum has broadened sufficiently, as it must do under the basic-flow straining, then turbulent effects begin to take over for $\kappa>\kappa_{\beta}$; figure 14 shows that they in fact dominate for $\kappa>20$.

The nature of the wave-mean-flow interaction is reflected in the spatial as well as in the spectral diagnostics. However, due to the nature of the initial conditions, the time-averaged spatial quantities for run $\mathrm{C}$ were rather noisy. Consequently, two other runs were performed that were identical with run $\mathrm{C}$ except for a different random specification of phases for the initial disturbance field (4.3), and an ensemble average of the three runs produced. The results are shown in figure 15 for $t=0-1,1-2$, and $0-15$. They are still not particularly smooth but at least the trends are clear.

The Reynolds-stress term $\langle\overline{u v}\rangle$ (figure 15a), the angle brackets here denoting a zonal and ensemble mean, is such as to transport momentum away from the westerly jet during $t=0-1$; this reflects the amplification of disturbance energy associated with eddy propagation into the westerly jet, and is in agreement with ray-tracing theory. But for $t=1-2$, and in the long time-average, the westerly jet is strengthened by the eddies, consistent with figure 13. The concentration of eddy amplitude in the westerly jet, and its initial meridional anisotropy, is also in agreement with 

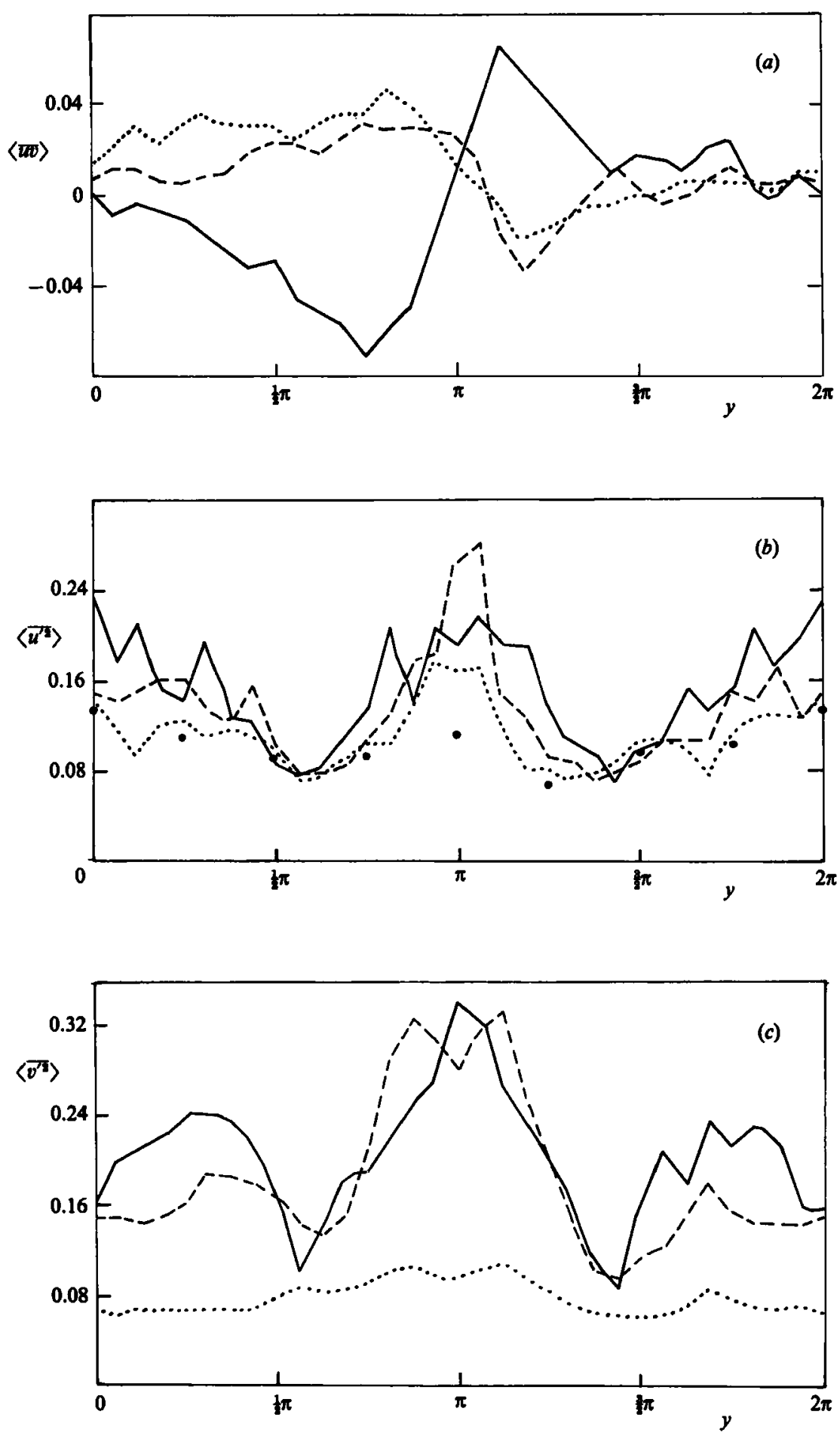

FIoURE 15. Ensemble average of $x$-averaged spatial diagnostics from run $C$ and two similar runs, time-averaged over $t=0-1(-), t=1-2(----)$, and $t=0-15(\cdots \cdots) \cdot(a)\langle\overline{u v}\rangle(y)$ (here the $t=0-15$ values have been multiplied by 5 to be visible); $(b)\left\langle\overline{u^{\prime 2}}\right\rangle(y) ;(c)\left\langle\overline{v^{\prime 2}}\right\rangle(y)$. The large dots in $(b)$ show, for selected $y$, the contribution from zonal-wave $(k \neq 0)$ modes for $t=0-15$. 
ray-tracing theory (figure $15 b, c)$. What is perhaps surprising is the intensity of eddy activity in the easterly jet (also evident in run A, e.g. figure $5 f$ ). This can however also be explained by appeal to ray theory, as disturbances originating in the easterly jet (for these parameter settings) have turning lines but not critical lines and are thus trapped in a waveguide; hence they do not decay by the mechanism of shear-induced transfer.

\subsection{Nonlinear, forced-dissipative equilibrium: run $\mathrm{D}$}

While spin-down runs have great pedagogical value, geophysical systemsparticularly the mid-latitude tropospheric transient eddy field - tend to be strongly forced and dissipated. Consequently it is important to determine whether the theoretical ideas advanced in $\$ 3$ can provide any insight into the behaviour of a model in forced-dissipative equilibrium. Here one such experiment, $\operatorname{run} D$, is examined in detail; the forcing and dissipation mechanisms are as described in \$4.1. Sensitivity to the external parameters is discussed briefly in $\S 5$. While the parameters have been chosen in $\$ 4.2$ to correspond as closely as possible to those of the atmosphere, the jet can only be considered 'realistic' insofar as it is zonal and of large scale relative to the disturbance.

The simulation extends over a period of $\Delta t=80$, and an ensemble of data is obtained by collecting samples taken every $\Delta t=0.2$ over the last $\Delta t=60$. For run $\mathrm{D}$ the turbulent eddy-turnaround time characterizing the smaller scales is about 0.1, so these samples can be considered independent for those scales; however the mixing timescale at the largest scales is considerably slower, on the order of $\Delta t=1$. To ensure that the statistics are reliable, the averaging period was divided into two segments of duration $\Delta t=30$ and the statistics of each compared ; no significant difference could be detected. Moreover another experiment, with a different arrangement of phases in the initial conditions, was performed and compared with run $\mathbf{D}$; again, no significant difference could be detected in the statistics. (This is not a trivial result, because the 'phase-locking' nature of the intermediate-scale forcing (4.6) implies that the initial conditions are not totally forgotten; the point is that the phases are nevertheless rapidly 'scrambled' by turbulence.)

Snapshots of the disturbance stream function and vorticity fields are shown in figure 16. These show that the turbulent activity is distributed inhomogeneously, with very little enstrophy present in the regions of strongest basic shear. On the largest scales of motion there is some evidence of meridional elongation, but the intermediate and smaller scales appear to be roughly isotropic - especially so when compared with features of similar size in run C (figure $8 b$ ).

The relative degree of anisotropy can be judged by an examination of figure 17, where the zonal and meridional spectra for run $D$ are compared with those for the relevant beta-plane turbulence experiment (i.e. with $U_{\mathrm{b}}=0$ ). The most notable difference between the figures is that in run $D$, the disturbance energy has penetrated significantly past the Rhines radius, up to the largest scales of motion; and, for $1<\kappa<\kappa_{\beta}$, the anisotropy is meridional rather than zonal. Between $\kappa_{\beta}$ and the forcing scale the flow is quite isotropic in both cases, but note that in run $D$ there is no concentration of energy at the forced scales themselves. This latter effect is presumably due to the effectiveness of the basic-flow straining in inducing spectral transfer on a relatively rapid timescale, but is somewhat surprising in view of the fact that the parameter $\epsilon$ of (3.6) is so small. Evidence of basic-flow straining can also be seen in the slight zonal anisotropy of the small scales.

It should be emphasized that while beta-plane turbulence does itself generate zonal 

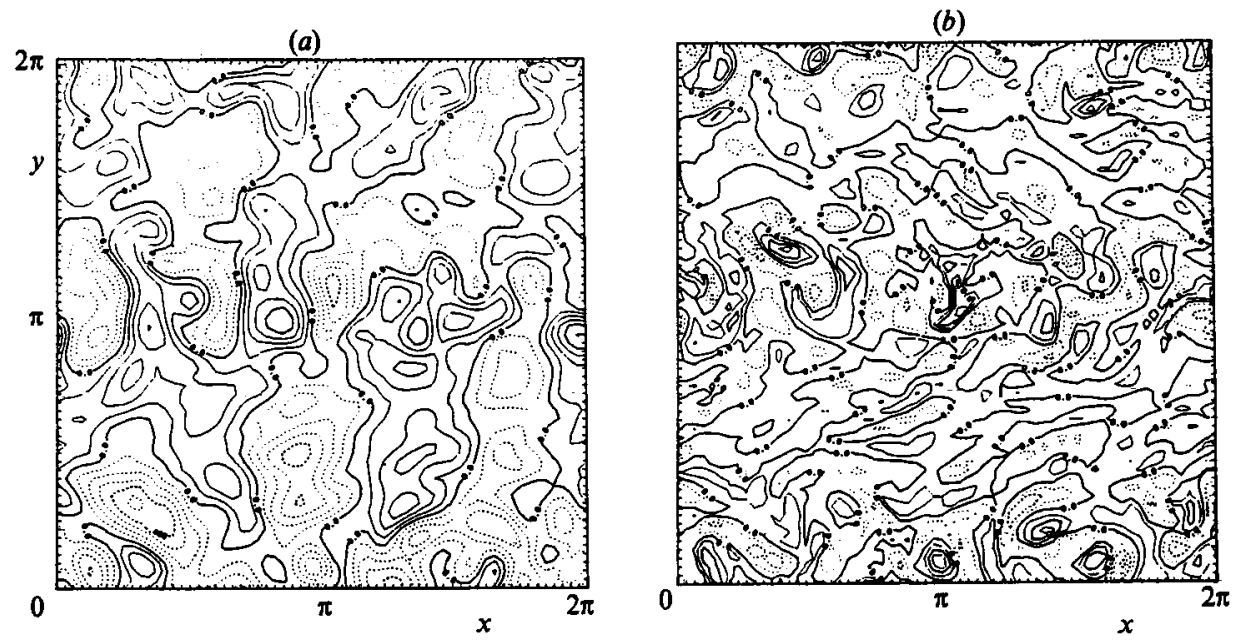

FiquRe 16. Instantaneous spatial maps during forced-dissipative equilibrium run $D$ of $(a)$ disturbance stream function $\phi(x, y)$ and $(b)$ disturbance vorticity $\nabla^{2} \phi(x, y)$.

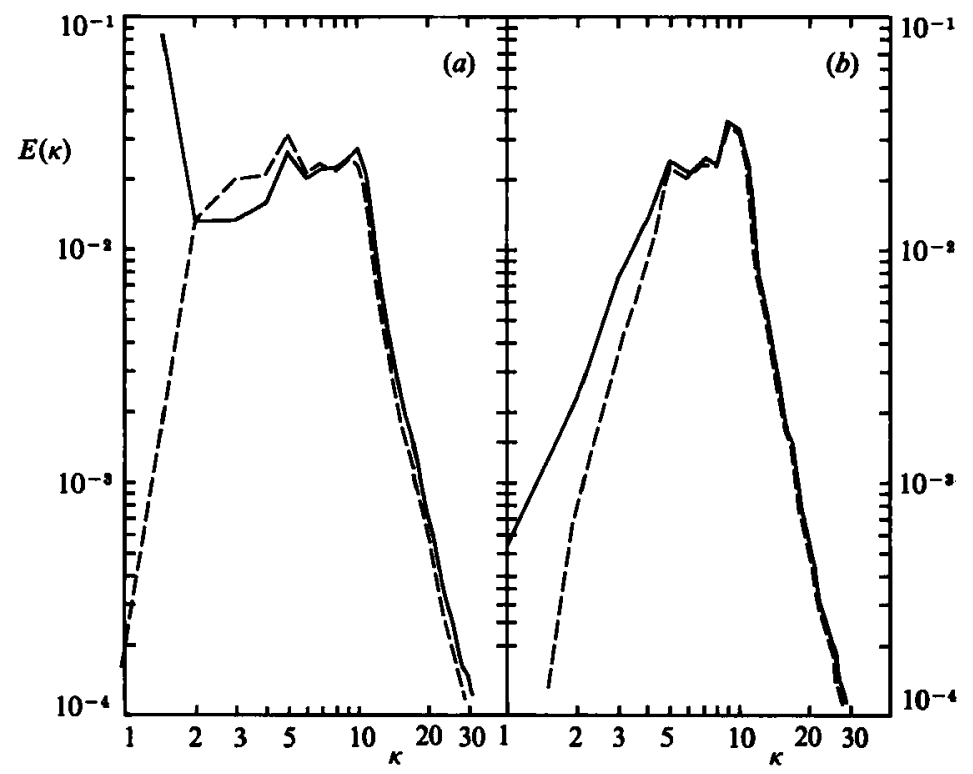

Figure 17. Time-averaged energy spectrum $E(\kappa)$ for $(a)$ run D, and $(b)$ an equivalent beta-plane turbulence experiment (i.e. no basic flow), decomposed into zonal $(\longrightarrow)$ and meridional (--- ) components.

jets, these tend to be relatively weak and to meander; thus the jets lead to shear-induced spectral transfer which is insignificant compared to that from the strong, phase-locked basic jets considered in this study. More will be said about this in $\$ 5$.

Examination of the two-dimensional $(k, l)$ spectra (not shown) reveals that the energy and enstrophy spectra associated with run D are (for $K>1$ ) approximately isotropic (in the sense that constant-energy contours tend to be aligned with curves 
of constant $\kappa$ ). The effect is much stronger than in run $\mathrm{C}$, for example, and is entirely absent in run $A$. This is consistent with the attribution of isotropization to irreversible turbulent dynamical mixing, as described in $\$ 3.3$ - indeed, the latter process is the only conceivable one that could effect isotropization.

Figure 18 shows the stationary-transient energy conversion terms, $C_{\mathrm{S}}(\kappa)$ and $C_{\mathrm{T}}(\kappa)$, for run $\mathrm{D}$, as well as the corresponding terms for enstrophy. As in run $\mathrm{C}, \kappa^{2} C_{\mathrm{T}}(\kappa)$ may evidently be viewed as a true interaction term for the transient flow. Unlike run $\mathrm{C}$, however, there is some net induced transfer of enstrophy from the forced scales to larger scales; with a forced-dissipative experiment, of course, one cannot know $a$ priori in which sense the time-averaged transfers will proceed. Figure $18(b)$ suggests that some of this initial up-scale transfer associated with intermediatescale forcing is reversed, but by no means all of it. Indeed, when viewed in terms of the energy (figure 18a), this net up-scale transfer is seen to be quite significant, penetrating to the largest scales of motion.

The fact that $C_{\mathrm{S}}(\kappa=1)$ is negative indicates a net transfer of energy from the stationary to the transient flow; this in turn implies that, to maintain equilibrium, the stationary flow must be forced rather than damped, which further implies equilibration of the $(k, l)=(0,1)$ mode below the amplitude of the basic flow. Such a result is not required, of course; equilibration could equally well have involved a maintenance of the $(0,1)$ mode above its basic-flow value. (Recall that the net result after 'spin-down' in run $\mathrm{C}$ was a strengthening of the basic-flow component; and see also figure 23 , where $F_{0}$ acts to damp the stationary flow.) In fact, the energy in the $(0,1)$ mode varied aperiodically within $2 \%$ of its mean value, but always kept below its initial value of 2.0. To test the robustness of this feature of the experiment, the basic-flow component was kicked by a factor of 1.08 at $t=20$, and allowed to re-equilibrate. Despite an energy level following the kick that was well in excess of its initial value, it rapidly fell back to its previous equilibrium range, and within $\Delta t=5$ this perturbed experiment was indistinguishable from run $\mathrm{D}$.

The relative importance of the processes of basic-flow straining and of nonlinear, turbulent transfer between different scales may be seen by looking at the relevant spectral fluxes of energy in figure 19. Evidently most of the spectral transfer is effected by the transient self-interaction term, which is to say by turbulence. The straining term is most important in removing energy from the forced intermediate scales and in transferring it non-locally up to the straining scales themselves. For $\kappa<5$ this is in fact the dominant term, but elsewhere the transient self-interaction term is stronger. Nevertheless, the significant differences between run D and classical beta-plane turbulence attest to the importance of basic-flow straining in determining the flow evolution.

One interesting feature of figure 19 that bears emphasis is the extent to which the 'induced' flux looks similar in form to the 'turbulent' flux. There is no reason why this has to be the case in general; here it results from the spectrally symmetric forcing and the degree of irreversibility in the induced up-scale transfers of enstrophy. Analysis in terms of $k$ and $l$ makes the dynamical distinction between the two different fluxes quite clear, but in this 'isotropic' representation the distinction is muddied.

Compared with the spin-down run $C$ (figure 14), run $D$ is characterized by a higher level of turbulence and the effects of shear-induced spectral transfer are correspondingly less visible. However these effects can still be isolated, provided one looks at the relevant diagnostic: namely the induced spectral transfers $C_{\mathrm{T}}(k ; l)$ and $\kappa^{2} C_{\mathrm{T}}(k ; l)$ considered as functions of $l$ for fixed $k$. According to the theory of $\S 3.2$, and as verified in the linear spin-down run (figure 6), the development of an initially 

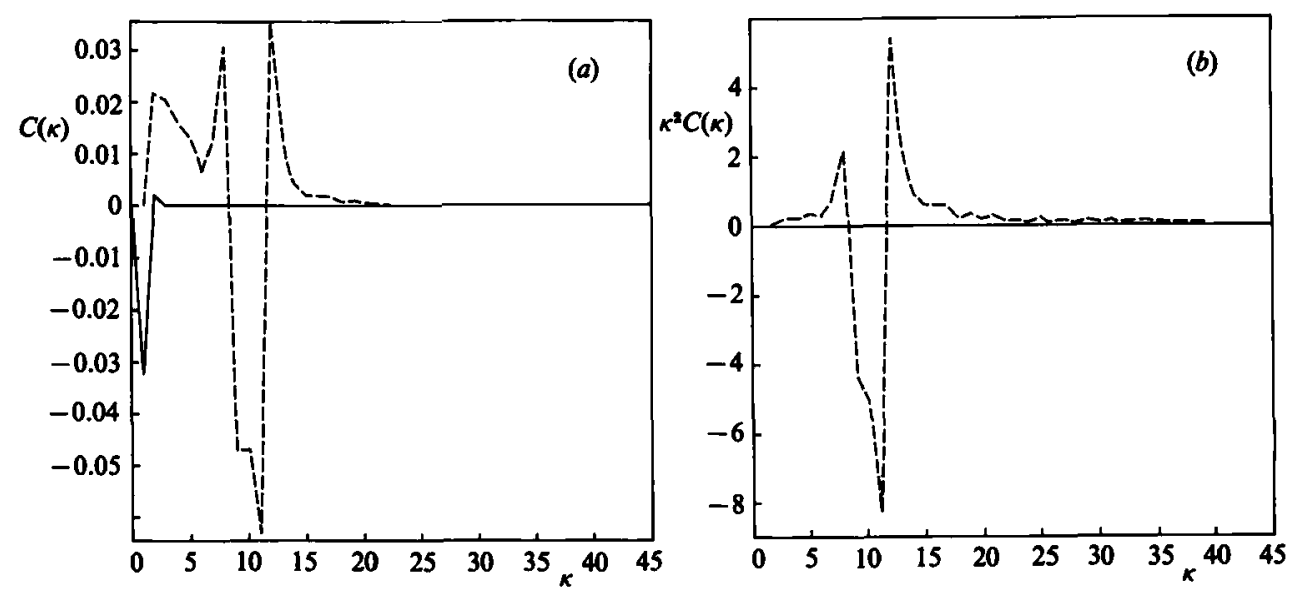

FIGURE 18. Time-averaged $(a)$ energy conversion terms $C_{\mathrm{S}}(\kappa)(-)$ and $C_{\mathrm{T}}(\kappa)(----)$, and $(b)$ enstrophy conversion terms $\kappa^{2} C_{\mathrm{S}}(\kappa)(-)$ and $\kappa^{2} C_{\mathrm{T}}(\kappa)(---)$, for run $\mathrm{D}$.

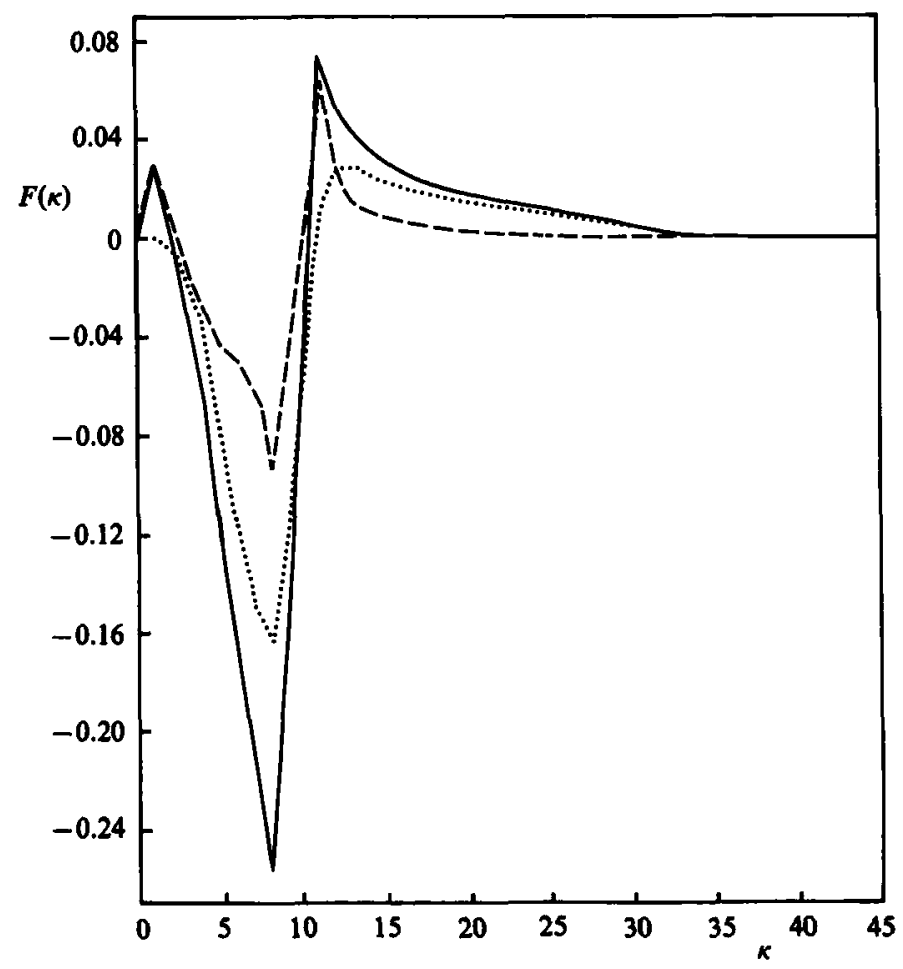

Figure 19. Time averaged spectral fluxes of energy, run $\mathrm{D}: F_{\mathrm{ST}}(\kappa)(----), F_{\mathrm{T}}(\kappa)(\cdots \cdots)$, and $F(\kappa)(\stackrel{)}{\longrightarrow}$.

spectrally isolated, symmetric disturbance involves both up-scale and down-scale induced transfer of enstrophy (the transfer being conservative to leading order in the scale separation factor $\gamma$ of (2.8)). But linear theory would always predict a reflection of the up-scale pulse. Consequently, what one should expect to see from these diagnostics in a time-averaged equilibrium situation is not evident $a$ priori.

What figure 20 shows, for $k=2$, is that on a time average, the shear-induced 


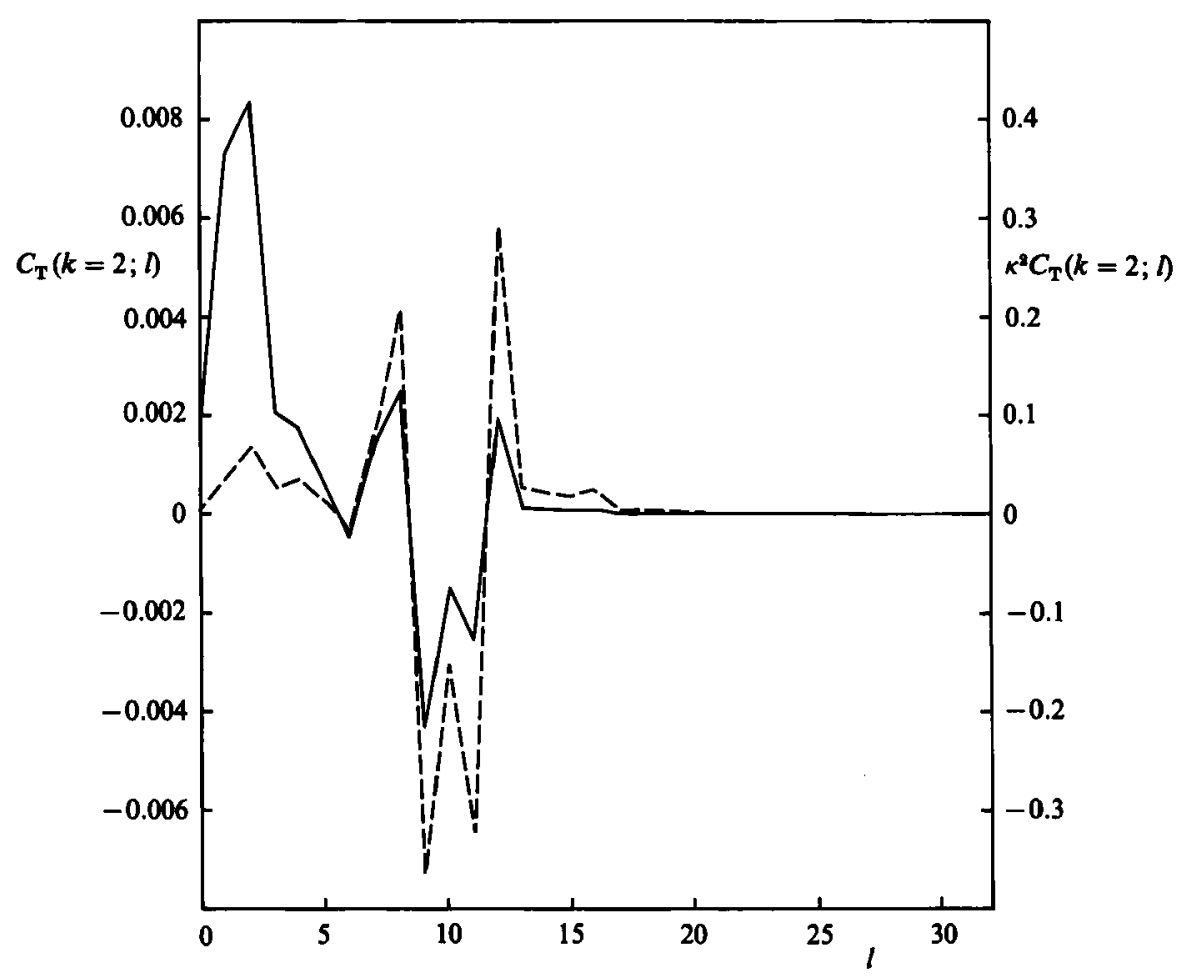

FiaURe 20. Time-averaged conversion terms of energy $C_{\mathrm{T}}(k ; l)(\longrightarrow)$ and of enstrophy $\kappa^{2} C_{\mathrm{T}}(k ; l)$ $(---)$, for fixed $k=2$ as functions of $l$, run $\mathrm{D}$.

spectral transfer is approximately symmetric about the forced scales; that is, about as much enstrophy gets transferred up-scale as down-scale. This leads, of course, to an amplification of the associated disturbance energy; indeed, it is the $k=2$ transient components that are the primary recipients of energy from the stationary flow. (Recall from \$3.2 that, all else being equal, amplification is strongest for lower- $k$ components.) Evidently the induced up-scale transfer is irreversible. Moreover the induced down-scale transfer is negligible for scales smaller than the forced ones; figure 19 shows that the enstrophy cascade is dominantly turbulent, even though it could in principle have been effected (as it was in run A) by the process of basic-flow straining.

In figure 20 the sum of $\kappa^{2} C_{\mathrm{T}}$ over all $l$ is less than $5 \%$ of the largest term. So even for this small value of $k$, with significant transfer involving $l=1,2$, the transient enstrophy is conserved to a good approximation in its interaction with the stationary flow. For larger $k$ the degree of conservation is even better, as one might expect.

The picture for $0<k<7$ is essentially like that of figure 20 , though with smaller amplitudes for $k \neq 2$; for $k>7$, on the other hand, the induced transfer is principally to higher $l$ (there is no room to move to smaller $l$ !), and the net effect of these scales is to strengthen the stationary flow. Overall, however, the effect from the smaller- $k$ waves dominates, and the stationary flow is a net source of transient energy. These results are summarized concisely in the quantities $C_{\mathrm{S}}(k)$ and $C_{\mathrm{T}}(k)$, shown in figure 21 ; the former indicates the net sense of the stationary-transient energetic interaction, while the latter demonstrates which $k$-scales gain energy and which lose it in the process. 


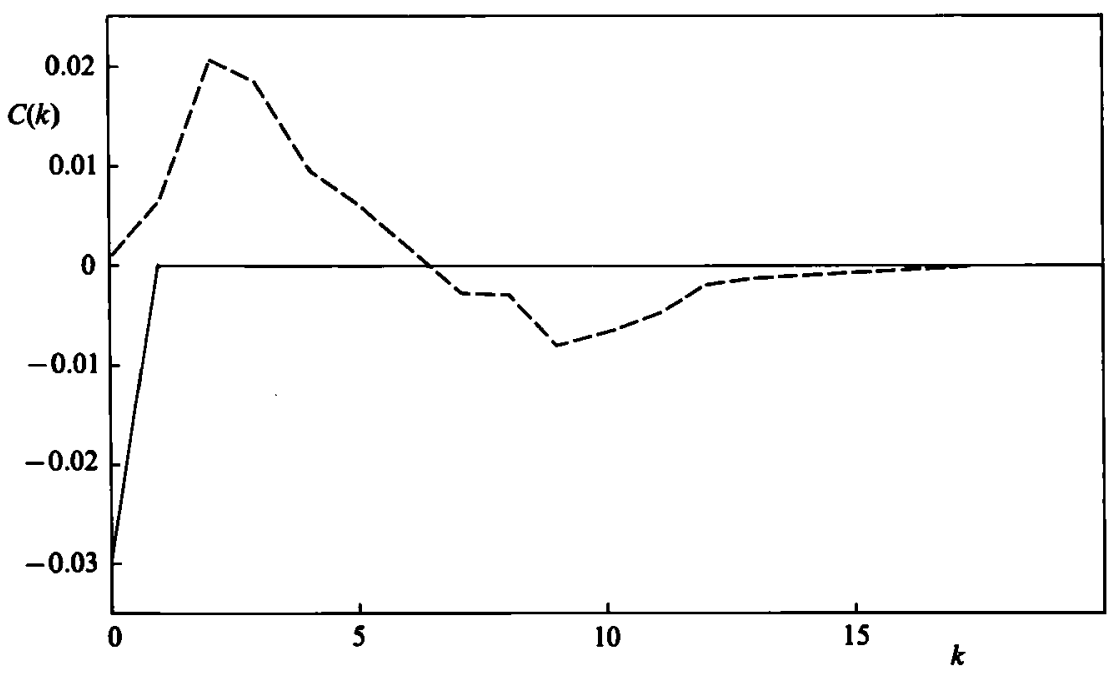

Figure 21. Time-averaged energy conversion terms $C_{\mathrm{S}}(k)(-)$ and $C_{\mathrm{T}}(k)(----)$, as functions of zonal wavenumber, for run $D$.

The time-averaged zonal-mean flow $(U+\langle\bar{u}\rangle)(y)$ (the angle brackets denoting a zonal mean) for run $\mathrm{D}$ is essentially indistinguishable by eye from the basic flow $U(y)$, when the two are plotted together. (Recall that the energy of the basic-flow $(k, l)=(0,1)$ component is only diminished by about $2 \%$ after equilibration.) However the nature of the wave-mean-flow interaction is clearly seen in the spatial diagnostics presented in figure 22(a,b). The Reynolds-stress term $\langle\overline{u v}\rangle$ (figure 22a) is such as to sharpen the westerly jet and broaden the easterly jet, roughly speaking, with the latter effect dominating to yield a net conversion of energy to the eddies. This result is quantified by figure $22(b)$, which depicts the two spatial forms of stationary-transient energy conversion analogous to $(2.5 c)$ (which is expressed in terms of the disturbance problem). Recall that in a straightforward manipulation of the momentum equation, $-(U+\langle\bar{u}\rangle) \mathrm{d}\langle\overline{u v}\rangle / \mathrm{d} y$ is the eddy-to-mean conversion term for the zonal-mean energy, while $-\langle\overline{u v}\rangle \mathrm{d}(U+\langle\bar{u}\rangle) / \mathrm{d} y$ is the mean-to-eddy conversion term for the eddy energy. Hence figure $22(b)$ shows that the eddies gain their energy from the regions of maximum zonal-mean shear, as one expects since that is where the mechanism of shear-induced spectral transfer is strongest. Finally, the spatial distribution of eddy energy is shown in figure $22(c)$ and evidently peaks in the jet cores, consistent with figure 16. The eddy energy appears roughly isotropic in the sense that $\left\langle\overline{u^{\prime 2}}\right\rangle \approx\left\langle\overline{v^{\prime 2}}\right\rangle$, but the $\left\langle\overline{u^{\prime 2}}\right\rangle$-term contains time deviations of the zonal-mean flow $\overline{\langle u\rangle^{\prime 2}}$; when this part is removed the remainder (indicated for selected values of $y$ by large dots in the figure) is somewhat less than $\left\langle\overline{v^{\prime 2}}\right\rangle$.

It might be argued that irreversibility of the shear-induced up-scale transfer could arise in forced-dissipative experiments from the Ekman damping. While large-scale damping must inevitably cause some irreversibility and could presumably account for the observed spectral transfer patterns, the accumulation of energy at large scales and the near-isotropic spectra cannot be explained in this way. Moreover the spin-down experiments, which are effectively inviscid at large scales, demonstrate clearly that nonlinearity is capable of effecting irreversibility of the shear-induced transfers. Of course, a consistent forced-dissipative balance could conceivably obtain without any spectral transfer whatsoever; in the event, however, the flow satisfies this balance nonlinearly. 

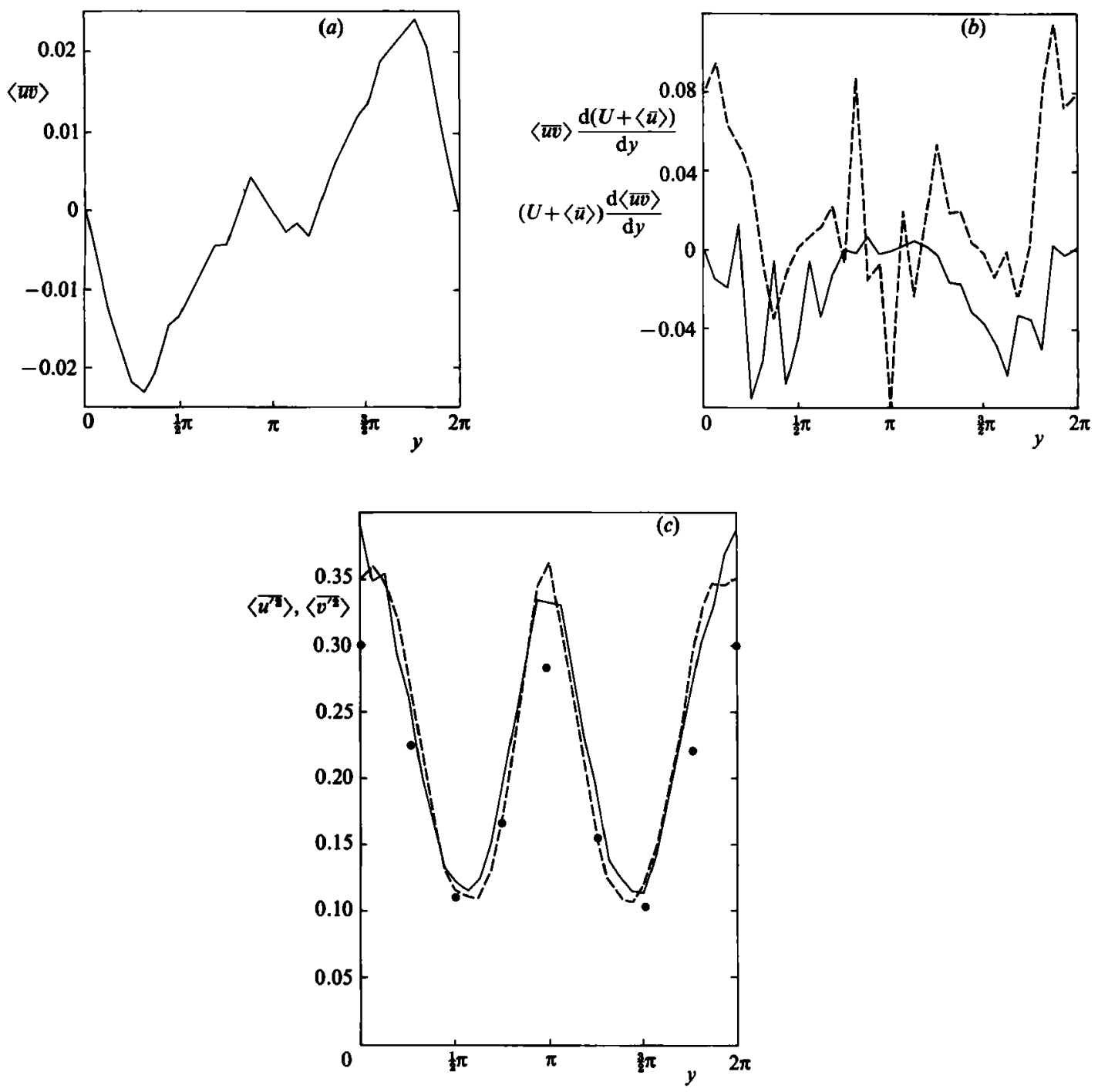

Fradre 22. Time- and zonal-averaged spatial diagnostics for run D. $(a)\langle\overline{u v}\rangle(y)$; (b) $\langle\overline{u v}\rangle \mathrm{d}(U+\langle\bar{u}\rangle) / \mathrm{d} y(-)$ and $(U+\langle\bar{u}\rangle) \mathrm{d}\langle\overline{u v}\rangle / \mathrm{d} y(--) ;(c)\left\langle\overline{u^{\prime 2}}\right\rangle(y)\left(\stackrel{-}{-}\right.$ and $\left\langle\overline{v^{\prime 2}}\right\rangle(y)$ $(---)$. The large dots in $(c)$ show, for selected $y$, the contribution to $\left\langle\overline{u^{\prime 2}}\right\rangle(y)$ from zonal-wave $(k \neq 0)$ modes.

\section{Discussion}

This study represents a partial attempt to extend the homogeneous theory of geostrophic turbulence into the inhomogeneous regime. In order to make some progress, attention has been focused on a particular problem of geophysical significance : namely that of barotropic beta-plane turbulence in the presence of a large-scale zonal jet. Then the inhomogeneity arises only from the mean flow, not from the fluid medium, and is restricted to the meridional coordinate; moreover it is of large scale. These simplifications enable a theoretical approach to be pursued, in which the transient disturbance dynamics may be understood as a combination of two distinct yet highly coupled processes. The first is an induced spectral transfer of disturbance 
enstrophy along lines of constant zonal wavenumber $k$, which corresponds physically to a straining of disturbance vorticity by the shear in the zonal basic flow. In this process the disturbance energy is generally not conserved, but the disturbance enstrophy is conserved to leading order in the scale separation parameter $\gamma$ of $(2.8)$.

The second process is one that might be called 'turbulent isotropization', and reflects the tendency of random nonlinear interactions to spread the enstrophy (and thereby the energy) along curves of constant total wavenumber $\kappa$. Insofar as this process - which will be effected by the transient disturbance self-interactions transfers enstrophy across lines of constant zonal wavenumber, it will interrupt and alter the phenomenon of basic-flow straining. Spectrally, one might view such interference as a 'scattering' which disrupts the delicate phase relations whose persistence is required to effect the shear-induced spectral transfer. Stated otherwise, shear-induced transfer is a systematic process that is vulnerable to disruption; whereas turbulent isotropization is a random process that indeed thrives on disruption. Consequently the latter will tend to dominate the former when the two effects operate on similar timescales.

The validity of this picture of the dynamics, and the way in which the two processes interact, has been examined by direct numerical simulation of the flow. It is found that the picture of classical beta-plane turbulence is altered, through the effect of the large-scale zonal flow, in the following ways: (i) while the turbulence is still confined to $\kappa>\kappa_{\beta}$, where $\kappa_{\beta}$ is Rhines's (1975) cascade-arrest scale, the disturbance penetrates strongly past $\kappa_{\beta}$ up to the largest scales of motion; (ii) the larger disturbance scales $\kappa<\kappa_{\beta}$ exhibit a tendency towards meridional rather than zonal anisotropy, in the sense that $v^{2}>u^{2}$ rather than vice versa; (iii) initial spectral transfers away from isotropic intermediate-scale forcing (or from an initial spike, in a spin-down experiment) are significantly enhanced by the shear-induced transfer associated with straining by the zonal flow, even though the scaling estimate of (3.6) would suggest otherwise. Indeed, when viewed in terms of $\kappa$ alone the shear-induced transfer away from the intermediate scales may frequently appear very similar to the conventional cascades of two-dimensional turbulence, even though the two processes are dynamically quite distinct. This may help account for the apparent success of twodimensional turbulence theory in describing the observed spectral fluxes (in terms of the total spherical harmonic wavenumber) of kinetic energy and enstrophy in the atmosphere (Boer \& Shepherd 1983; Shepherd 1987b).

Seen from the opposite perspective, the shear-induced spectral transfer obtained in the context of linearized theory-meaning with disturbance self-interactions suppressed - is altered, by full nonlinearity, in the following ways: (i) the up-scale transfer of disturbance enstrophy is made into a net, that is to say irreversible, transfer, rather than being purely temporary; (ii) the down-scale transfer is superseded very quickly by the (slower) turbulent transfer, so that basic-flow straining has little systematic effect on the smaller scales of motion; (iii) tendencies to anisotropy are mitigated. Shear-induced spectral transfer of disturbance enstrophy remains the mechanism by which the wave-mean-flow interaction may be most directly understood; the point is that the closure of the interaction problem, and the determination of whether the disturbance acts to weaken or to strengthen the basic flow, is crucially dependent upon strongly nonlinear, turbulent effects.

Indeed this latter aspect of the problem was found to be quite sensitive to changes in the non-dimensional parameters in parallel forced-dissipative experiments (although the conclusions presented above were all robust to such changes); for a more complete description one may refer to Shepherd (1984, ch. VIII). These 
experiments all showed a distinct pattern : for small but non-zero zonal wavenumber $k$, the induced transfer is spectrally symmetric in $l$ about the input scale, and leads to a net up-scale energy transfer and amplification at the expense of the stationary flow; while for intermediate $k$ the transfer process is essentially down-scale, implying absorption of transient energy into the stationary flow and a strengthening of the basic-flow mode. The net sense of the stationary-transient interaction depends sensitively on the position of the transition scale $k_{\mathrm{t}}$ between these two regimes.

For example, when the value of $\beta_{0}$ was increased from 25 to 64 , moving $\kappa_{\beta}$ out to $\kappa_{\beta} \approx 8$, the net conversion from stationary to transient energy increased by about $50 \%$, even though $k_{\mathrm{t}}$ only moved from $k=6$ to $k=7$. On the other hand, when the disturbance energy was doubled (necessitating a halving of the Ekman damping coefficient $r$ to maintain stationarity), the net conversion changed sign and $k_{\mathrm{t}}$ was reduced to $k=4$. This change is clearly seen in figure 23 , which shows $C_{\mathrm{S}}(k)$ and $C_{\mathrm{T}}(k)$ from this run and is to be compared with figure 21. Neither of these changes in $k_{\mathrm{t}}$ would appear to be a simple consequence of the parametric alterations; indeed, one might have expected that doubling $E(\phi)$ would have decreased the importance of the 'linear' straining dynamics and would therefore have weakened the forcing of the basic flow, but in fact the opposite occurred. This suggests that the coupling between the essential dynamical processes of shear-induced spectral transfer and turbulent isotropization is generally rather complicated, and that quantitative closure may well need to be correspondingly sophisticated if one desires a parameterization of the transient dynamics in terms of the stationary flow. But it is hoped that the diagnostic utility of the theoretical ideas advanced here for the understanding of strongly nonlinear inhomogeneous flow has now been established.

Indeed, the essentially non-local character of shear-induced transfer, and its sensitivity to turbulent effects, imply that attempts to model stationary-transient interaction using a severely truncated or, even worse, a linear model are doomed to failure. Clearly a wide range of interacting transient scales of motion needs to be considered in such studies. However, the fact that turbulent activity can limit the range of significant stationary-transient interaction, as has been demonstrated, means that one might hope to parameterize the dynamics beyond this range using a reasonably sophisticated turbulent closure (e.g. Sadourny \& Basdevant 1985) in situations where the level of nonlinearity is sufficiently high. Interpretation of atmospheric data in the light of these ideas, which provides an estimate of the degree of non-localness of tropospheric stationary-transient interactions, is presented elsewhere (Shepherd $1987 b, c)$.

Although the emphasis of this study has been on spectral diagnostic approaches, a few remarks are in order concerning the spatial signature of the stationary-transient interactions seen in forced-dissipative experiments. The robust results are that the eddies (defined relative to the time- and zonal-average flow) gain energy from the regions of strongest mean shear; the easterly jet is weakened; and the westerly jet is sharpened (e.g. figure 22 for run $D$ ). The latter effect seems similar to the barotropic eddy forcing of the westerly jet found in the modelling study of McWilliams \& Chow (1981). All of these points may be understood in terms of the linear theory of mean-flow straining presented in \$3.2. What is not robust is however the relative importance of these effects, and such parameter dependence of the net mean-eddy interaction is of course intimately linked to the parallel sensitivity of the net stationary-transient interaction discussed above. As regards the eddy variances, they were always found to exhibit maxima in the jet cores and minima in the strongly sheared regions. Where the eddy variances were anisotropic, the anisotropy was 


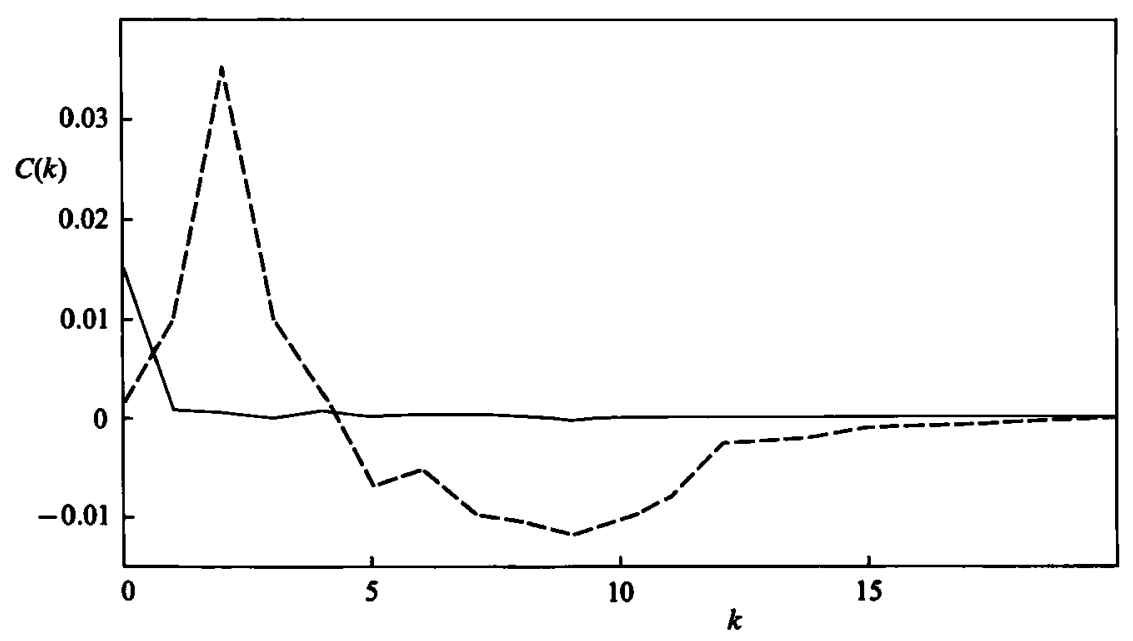

Figure 23. Time-averaged energy conversion terms $C_{\mathrm{S}}(k)(-)$ and $C_{\mathrm{T}}(k)(---)$, as functions of zonal wavenumber, for forced-dissipative equilibrium run similar to run $\mathbf{D}$ but with disturbance energy doubled and linear damping coefficient halved.

always meridional rather than zonal. Such concentration of eddy variance in jet core regions was also found by McWilliams \& Chow (1981), and for the case presented in their figure 5 the eddies showed strong meridional anisotropy. It seems likely that the present work might help explain their results.

The weakness of the net wave-mean-flow interaction, and its sensitivity to the run parameters, are direct consequences of turbulent isotropization of the transient eddy field: near-isotropy of smaller scales imposes a constraint on the triad interactions which precludes a strong large-scale barotropic response (Pedlosky 1962). If this constraint were removed, for instance by localizing the disturbance forcing and the regions of strong nonlinearity, then the net interaction could be significant (Lorenz 1953); an example of such barotropic driving by inhomogeneous forcing is given by Rhines $(1977, \S 8 \mathrm{C})$. Such effects are certainly worth investigating in future work along these lines.

In this study much emphasis has been placed on the differences between the present dynamical regime and that of beta-plane turbulence. But insofar as beta-plane turbulence generates its own zonal jets internally, one is naturally led to wonder why these zonally elongated eddies do not induce large-scale meridional anisotropy and penetration of transient energy past the 'Rhines radius' $\kappa=\kappa_{\beta}$, as the large-scale forced jet does here. The simple answer is that the jets generated in beta-plane turbulence tend to be relatively weak and to meander around the domain; thus they are not capable of effecting systematic straining to the extent that a phase-locked, large-amplitude jet is. In fact one can identify the shear-induced spectral transfer associated with the beta-jets in the numerical experiments, but it is of an insignificant magnitude.

But there is perhaps a more fundamental reason why beta-jets cannot induce significant spectral transfer past $\kappa_{\beta}$, which is that disturbances with $\kappa<\kappa_{\beta}$ are probably unstable with respect to the $k=0, l \approx \kappa_{\beta}$ modes. While there is no clear theoretical basis for this result (there are two obstacles: one is that the hypothesized perturbation is of comparable magnitude with the 'basic flow'; the other is that strict application of Rossby-wave resonant-interaction theory forbids interaction with 
$k=0$ modes), the numerical evidence seems incontrovertible. In the first place there is the experience from beta-plane turbulence itself. In addition, a number of experiments were performed with the present model using basic jets with smaller-scale meridional structure (details are given in Shepherd 1984, Appendix, and Shepherd $1987 c$ ). Regardless of whether an $l=1$ component also existed which might induce transfer up to the largest scales of motion, as in the experiments shown here, it was found in all cases that there was no significant penetration of disturbance energy past the isotropic circle with radius equal to the smallest scale of the zonal jet. Applied to the beta-jets, it may be concluded that even if they were locked in position, purely zonal, and strong, they would still be unable to induce spectral transfer past $\kappa=\kappa_{\beta}$. This is an intriguing result which certainly demands further investigation. However, it is well beyond the scope of the present study, because it requires a theory that can treat the interactions between a stationary flow and transient disturbances of larger as well as of smaller scale.

The present research represents part of the author's Ph.D. dissertation, which was completed at the Massachusetts Institute of Technology; he is especially grateful to his thesis supervisor, Peter Rhines, for much critical advice, as well as to Greg Holloway and Michael McIntyre for subsequent helpful discussions. Comments from the referees were also much appreciated. The basic spectral code for the numerical simulations was kindly provided by Dale Haidvogel, and the simulations were run on the CRAY-1 computer at the National Center for Atmospheric Research in Boulder. (NCAR is sponsored by the National Science Foundation.) The author would also like to thank the Oceanography Section at NCAR for making possible a lengthy summer visit during which many of the key ideas in this work crystallized. Funding has been provided at various times by NSF grants ATM-76-20070, ATM-81-15712, and OCE-82-19780; by NASA grant NGR-22-009-727; and by fellowships from the Natural Sciences and Engineering Research Council of Canada. The hospitality of the Department of Applied Mathematics and Theoretical Physics, Cambridge University, and support from St Catharine's College, Cambridge, and the UK Natural Environment Research Council, during the writing of this paper is gratefully acknowledged.

\section{Appendix}

The spectral diagnostic quantities of $\$ \S 2.3$ and 4.5 are defined as follows: (here the curly brackets as well as the circumflex indicate a Fourier transform)

$$
\hat{A}(\boldsymbol{\kappa}) \equiv \iint A(x, y) \mathrm{e}^{-\mathrm{i}(k x+l y)} \mathrm{d} x \mathrm{~d} y \equiv\{A\}(\boldsymbol{\kappa}) .
$$

For the disturbance/basic-flow decomposition, where $\phi$ is the disturbance stream function and $\Psi$ that of the basic flow,

$$
\begin{gathered}
E_{\mathrm{D}}(\boldsymbol{\kappa}) \equiv \frac{1}{2} \kappa^{2}|\hat{\phi}(\boldsymbol{\kappa})|^{2} ; \quad G_{\mathrm{D}}(\boldsymbol{\kappa}) \equiv \boldsymbol{\kappa}^{2} E_{\mathrm{D}}(\boldsymbol{\kappa}) ; \\
I_{\mathbf{D}}(\boldsymbol{\kappa}) \equiv \frac{1}{2} \hat{\phi}^{*}(\boldsymbol{\kappa})\left\{\mathrm{J}\left(\phi, \nabla^{2} \phi\right)\right\}(\boldsymbol{\kappa})+\text { c.c. } \\
C_{\mathrm{D}}(\boldsymbol{\kappa}) \equiv \frac{1}{2} \hat{\phi}^{*}(\boldsymbol{\kappa})\left[\left\{\mathrm{J}\left(\Psi, \nabla^{2} \phi\right)\right\}(\boldsymbol{\kappa})+\left\{\mathrm{J}\left(\phi, \nabla^{2} \Psi\right)\right\}(\boldsymbol{\kappa})\right]+\text { c.c. } \\
D_{\mathbf{D}}(\boldsymbol{\kappa}) \equiv-\frac{1}{2} \hat{\phi}^{*}(\boldsymbol{\kappa})\{\mathrm{S}(\phi)\}(\boldsymbol{\kappa})+\text { c.c. }
\end{gathered}
$$


For the stationary/transient decomposition, where $\bar{\psi}$ is the time-mean stream function over some given interval and $\psi^{\prime}$ the deviation therefrom,

$$
\begin{gathered}
E_{\mathrm{T}}(\boldsymbol{\kappa}) \equiv \frac{1}{2} \kappa^{2} \overline{\left.\psi^{\prime}(\boldsymbol{\kappa})\right|^{2}} ; \quad G_{\mathrm{T}}(\boldsymbol{\kappa}) \equiv \kappa^{2} E_{\mathrm{T}}(\boldsymbol{\kappa}) ; \\
I_{\mathrm{T}}(\boldsymbol{\kappa}) \equiv \frac{1}{2} \hat{\psi}^{\prime *}(\boldsymbol{\kappa})\left\{\mathrm{J}\left(\psi^{\prime}, \nabla^{2} \psi^{\prime}\right)\right\}(\boldsymbol{\kappa})+\text { c.c. } \\
C_{\mathrm{T}}(\boldsymbol{\kappa}) \equiv \frac{1}{2} \overline{\psi^{\prime *}(\boldsymbol{\kappa})\left[\left\{\mathrm{J}\left(\bar{\psi}, \nabla^{2} \psi^{\prime}\right)\right\}(\boldsymbol{\kappa})+\left\{\mathrm{J}\left(\psi^{\prime}, \nabla^{2} \bar{\psi}\right)\right\}(\boldsymbol{\kappa})\right]}+\text { c.c. } \\
D_{\mathrm{T}}(\boldsymbol{\kappa}) \equiv-\frac{1}{2} \overline{\psi^{\prime *}(\boldsymbol{\kappa})\left\{\mathbf{S}^{\prime}(\psi)\right\}(\boldsymbol{\kappa})}+\text { c.c. }
\end{gathered}
$$

while

$$
\begin{aligned}
& E_{\mathrm{S}}(\boldsymbol{\kappa}) \equiv \frac{1}{2} \kappa^{2}|\overline{\hat{\psi}}(\kappa)|^{2} ; \quad G_{\mathrm{S}}(\kappa) \equiv \kappa^{2} E_{\mathrm{S}}(\kappa) ; \\
& I_{\mathrm{S}}(\boldsymbol{\kappa}) \equiv \frac{1}{2} \bar{\psi}^{*}(\boldsymbol{\kappa})\left\{\mathrm{J}\left(\bar{\psi}, \nabla^{2} \bar{\psi}\right)\right\}(\boldsymbol{\kappa})+\text { c.c. } ; \\
& C_{\mathrm{S}}(\boldsymbol{\kappa}) \equiv \frac{1}{2} \overline{\hat{\psi}}^{*}(\boldsymbol{\kappa})\left\{\overline{\mathrm{J}\left(\psi^{\prime}, \nabla^{2} \psi^{\prime}\right)}\right\}(\boldsymbol{\kappa})+\text { c.c. } ; \\
& D_{\mathrm{S}}(\boldsymbol{\kappa}) \equiv-\frac{1}{2} \overline{\hat{\psi}}^{*}(\boldsymbol{\kappa})\{\overline{\mathrm{S}}(\psi)\}(\boldsymbol{\kappa})+\text { c.c. }
\end{aligned}
$$

\section{REFERENCES}

ANDREws, D. G. 1983 A conservation law for small-amplitude quasi-geostrophic disturbances on a zonally asymmetric basic flow. J. Atmos. Sci. 40, 85-90.

Andrews, D. G. \& McIntyre, M. E. 1976 Planetary waves in horizontal and vertical shear: The generalized Eliassen-Palm relation and the mean zonal acceleration. J. Atmos. Sci. 33, 2031-2048.

Basdevant, C., Legras, B., Sadourny, R. \& BÉland, M. 1981 A study of barotropic model flows: Intermittency, waves and predictability. J. Atmos. Sci. 38, 2305-2326.

Basdevant, C. \& Sadourny, R. 1983 Modélisation des échelles virtuelles dans la simulation numérique des écoulements turbulents bidimensionnels. J. Méc. Théor. Appl. 2, numéro spécial, 243-269.

Batchelor, G. K. 1953 The Theory of Homogeneous Turbulence. Cambridge University Press, $197 \mathrm{pp}$.

Batchelor, G. K. 1969 Computation of the energy spectrum in homogeneous two-dimensional turbulence. Phys. Fluids 12, Suppl. II, 233-239.

Bennett, A. F. \& Haidvoare, D. B. 1983 Low-resolution numerical simulation of decaying two-dimensional turbulence. J. Atmos. Sci. 40, 738-748.

Boer, G. J. \& SHEPherd, T. G. 1983 Large-scale two-dimensional turbulence in the atmosphere. J. Atmos. Sci. 40, 164-184.

Booker, J. R. \& Bretherton, F. P. 1967 The critical layer for internal gravity waves in a shear flow. J. Fluid Mech. 27, 513-539.

Bretherton, F. P. \& Garrett, C. J. R. 1968 Wavetrains in inhomogeneous moving media. Proc. $R$. Soc. Lond. A 302, 529-554.

Bretherton, F. P. \& Haidvogel, D. B. 1976 Two-dimensional turbulence above topography. J. Fluid Mech. 78, 129-154.

Carnevale, G. F. 1982 Statistical features of the evolution of two-dimensional turbulence. J. Fluid Mech. 122, 143-153.

Charney, J. G. 1971 Geostrophic turbulence. J. Atmos. Sci. 28, 1087-1095.

Chen, T.-C. \& Wins-Nielsen, A. C. 1978 Non-linear cascades of atmospheric energy and enstrophy in a two-dimensional spectral index. Tellus 30, 313-322.

Farge, M. \& SADouray, R. 1986 Inhibition de la turbulence bidimensionnelle par une rotation d'entrainement. C. R. Acad. Sci. Paris 302, Série II, 847-850.

FJørtort, R. 1953 On the changes in the spectral distribution of kinetic energy for two dimensional, nondivergent flow. Tellus 5, 225-230. 
Fox, D. G. \& Orszag, S. A. 1973 Pseudospectral approximation to two-dimensional turbulence. J. Comp. Phys. 11, 612-619.

Haidvogel, D. B. \& Held, I. M. 1980 Homogeneous quasi-geostrophic turbulence driven by a uniform temperature gradient. J. Atmos. Sci. 37, 2644-2660.

HELD, I. M. 1983 Stationary and quasi-stationary eddies in the extratropical troposphere: Theory. In Large-Scale Dynamical Processes in the Atmosphere (ed. B. J. Hoskins \& R. E. Pearce), pp. 127-168. Academic.

HeLd, I. M. 1985 Pseudomomentum and the orthogonality of modes in shear flows. J. Atmos. Sci. 42, 2280-2288.

Herring, J. R. 1975 Theory of two-dimensional anisotropic turbulence. J. Atmos. Sci. 32, 2254-2271.

Herring, J. R. 1977 Two-dimensional topographic turbulence. J. Atmos. Sci. 34, 1731-1750.

HolLOWaY, G. 1978 A spectral theory of nonlinear barotropic motion above irregular topography. J. Phys. Oceanogr. 8, 414-427.

HolLoway, G. 1983 Effects of planetary wave propagation and finite depth on the predictability of atmospheres. J. Atmos. Sci. 40, 314-327.

Holloway, G. \& Hendershott, M. C. 1977 Stochastic closure for nonlinear Rossby waves. J. Fluid Mech. 82, 747-765.

JEFFREYS, H. 1933 The function of cyclones in the general circulation. Procès-Verbaux de l'Association de Météorologie, UGGI (Lisbon). Reprinted in Theory of Thermal Convection (ed. B. Saltzman), pp. 200-211. Dover (1962).

Kraichnan, R. H. 1967 Inertial ranges in two-dimensional turbulence. Phys. Fluids 10 , $1417-1423$.

Legras, B. 1980 Turbulent phase shift of Rossby waves. Geophys. Astrophys. Fluid Dym. 15, 253-281.

Leith, C. E. 1968 Diffusion approximation for two-dimensional turbulence. Phys. Fluids 11, 671-673.

LighthiLl, M. J. 1978 Waves in Fluids. Cambridge University Press, 504 pp.

LIN, H. 1982 Weakly inhomogeneous turbulence theory with applications to geophysical flows. $\mathrm{Ph} . \mathrm{D}$. thesis, Massachusetts Institute of Technology, $146 \mathrm{pp}$. (also NCAR Cooperative Thesis NCAR/CT-69).

LoREnz, E. N. 1953 The interaction between a mean flow and random disturbances. Tellus 5, 238-250.

LoREnz, E. N. 1960 Maximum simplification of the dynamic equations. Tellus 12, 243-254.

MoWritiams, J. C. 1984 The emergence of isolated coherent vortices in turbulent flow. J. Fluid Mech. 146, 21-43.

MoWriliams, J. C. \& Chow, J. H. S. 1981 Equilibrium geostrophic turbulence I: A reference solution in a beta-plane channel. J. Phys. Oceanogr. 11, 921-949.

MERILEES, P. E. \& WARN, H. 1975 On energy and enstrophy exchanges in two-dimensional non-divergent flow. J. Fluid Mech. 69, 625-630.

Möhring, W. 1978 Acoustic energy flux in nonhomogeneous ducts. J. Acoust. Soc. Am. 64, 1186-1189.

ORR, W. M'F. 1907 The stability or instability of the steady motions of a perfect liquid and of a viscous liquid. Parts I and II. Proc. R. Irish Acad. A 27, 9-68; 69-138.

Pedlosky, J. 1962 Spectral considerations in two-dimensional incompressible flow. Tellus 14, 125-132.

Phillits, O. M. 1977 The Dynamics of the Upper Ocean, 2nd edn. Cambridge University Press, $336 \mathrm{pp}$.

Rhines, P. B. 1975 Waves and turbulence on a beta-plane. J. Fluid Mech. 69, 417-443.

Rhines, P. B. 1977 The dynamics of unsteady currents. In The Sea, vol. 6 (ed. E. D. Goldberg, I. N. McCave, J. J. O'Brien \& J. H. Steele), pp. 189-318. Wiley

Rhines, P. B. 1979 Geostrophic turbulence. Ann. Rev. Fluid Mech. 11, 401-441.

Sadourny, R. 1985 Quasi-geostrophic turbulence: An introduction. In Turbulence and Predictability in Geophysical Fluid Dynamics and Climate Dynamics (ed. M. Ghil, R. Benzi \& G. Parisi), Corso LXXXVIII, pp. 133-158. North-Holland. 
Sadourny, R. \& Basdevant, C. 1985 Parameterization of subgrid scale barotropic and baroclinic eddies in quasi-geostrophic models: Anticipated potential vorticity method. J. Atmos. Sci.42, $1353-1363$.

Salmon, R. 1978 Two-layer quasi-geostrophic turbulence in a simple special case. Geophys. Astrophys. Fluid Dyn. 10, 25-52.

Salmon, R. 1980 Baroclinic instability and geostrophic turbulence. Geophys. Astrophys. Fluid Dyn. 12, 167-211.

Salmon, R. 1982 Geostrophic turbulence. In Topics in Ocean Physics (ed. A. R. Osborne \& P. Malanotte-Rizzoli), Corso LXXX, pp. 30-78. North-Holland.

Shepherd, T.G. 1984 Rossby waves and two-dimensional turbulence in the presence of a large-scale zonal jet. Ph.D. thesis, Massachusetts Institute of Technology, 393 pp.

Shepherd, T. G. 1985 Time development of small disturbances to plane Couette flow. J. Atmos. Sci. 42, 1868-1871.

SHEPHERD, T. G. 1987 a Non-ergodicity of inviscid two-dimensional flow on a beta-plane and on the surface of a rotating sphere. J. Fluid Mech. 184, 289-302.

Shepherd, T. G. $1987 b$ A spectral view of nonlinear fluxes and stationary-transient interaction in the atmosphere. J. Atmos. Sci. 44, 1166-1178.

ShePherd, T. G. $1987 c$ Inhomogeneous two-dimensional turbulence in the atmosphere. In Proc. 1986 European Turbulence Conference (ed. J. Mathieu \& G. Comte-Bellot). Springer (to appear).

Tang, C.-M. \& Orszag, S. A. 1978 Two-dimensional turbulence on the surface of a sphere. J. Fluid Mech. 87, 305-319.

Tномson, W. 1887 Stability of fluid motion - rectilineal motion of viscous fluid between two parallel planes. Phil. Mag. 24, 188-196.

Tung, K. K. 1983 Initial-value problems for Rossby waves in a shear flow with critical level. J. Fluid Mech. 133, 443-469. 